$$
\text { كلية الإدارة والاقتصاد -جامعة الموصل }
$$

$$
\text { المستخلص }
$$

من مقومسات نمو وتقدم أي شركة أو مؤسسة تجاريـة الاستعانة بأسـاليب علميـة حديثة

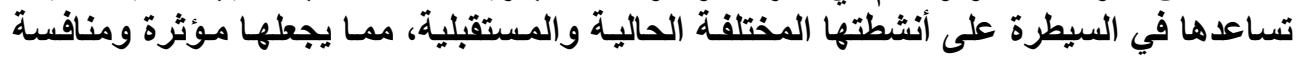

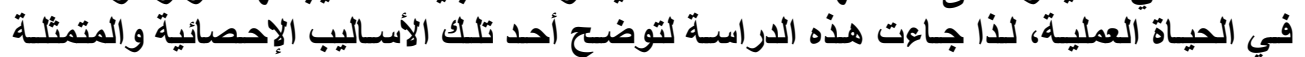

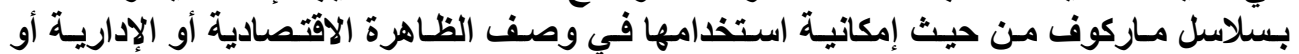

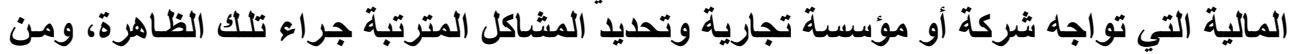

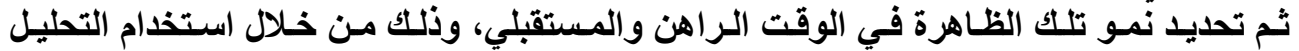

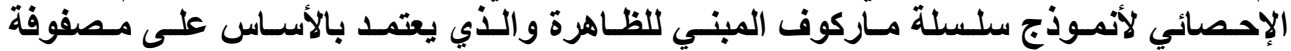

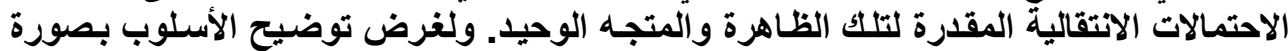

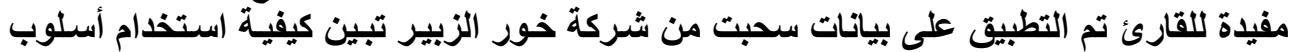

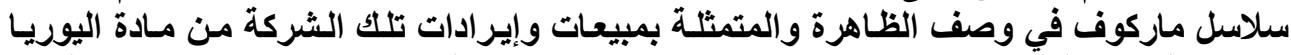

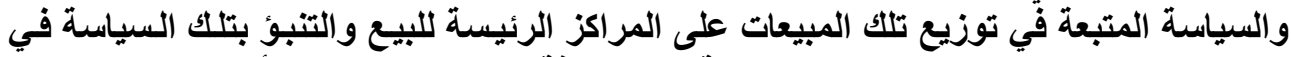

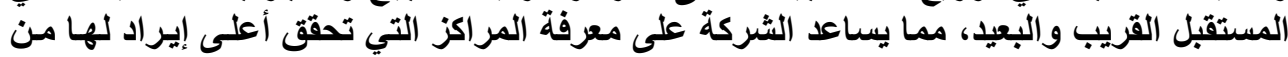

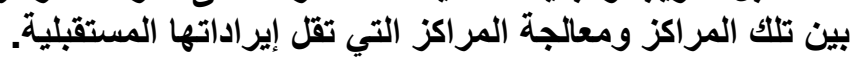

\title{
Markov Chains between Theory and Application In the Economic, Administrative or Financial Field
}

\author{
Rikan A.A. Ahmed \\ Lecturer \\ Department of Statistics \\ University of Basra
}

\begin{abstract}
One of the principles of the development or improvement of any company or commercial establishment is demanded by the help of new or modern scientific which can help it to control its different activities whether in the present or in the future. This makes it affective and competent in the business. The study above shows one of these methods which are clearly which are clearly with




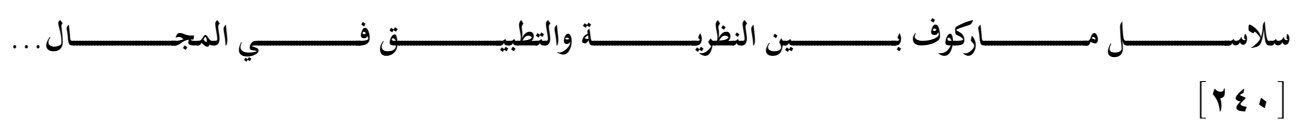

Markov chains, especially in the possibility of its use when we want to describe the economic, administrative and financial phenomenon etc for the company or the commercial establishment. Markov chains can be used to state the problems which the company is faced and it can determine the development of these phenomena in the present and in the future by using statistical analysis for this chain in the phenomenon.

المقدمة

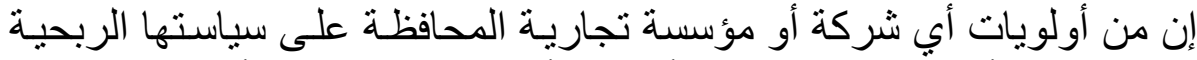

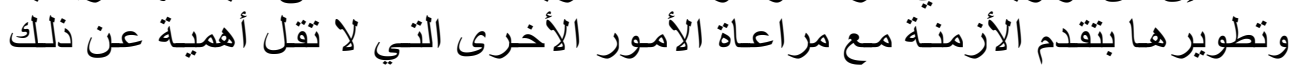

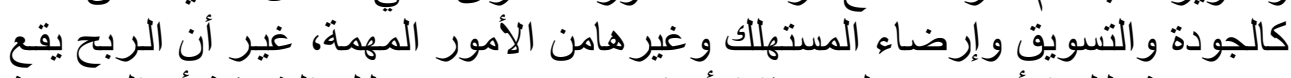

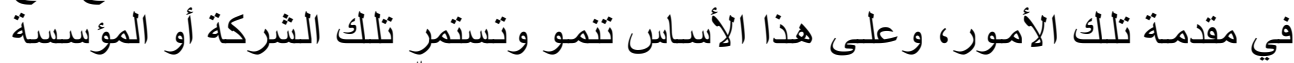

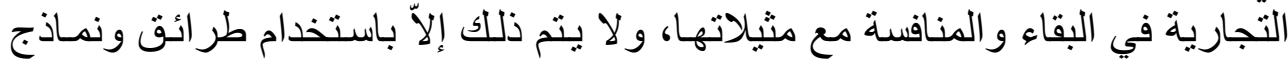

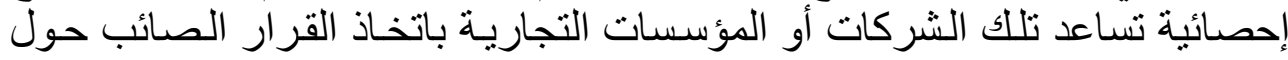

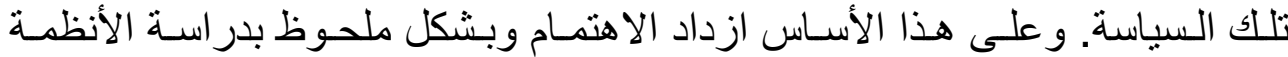

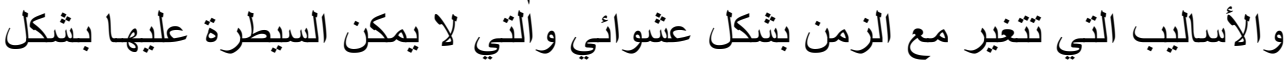

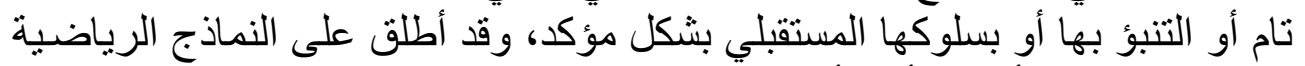
التي تمثل هذه الأنظمـة أو الأسـاليب بالعمليات التصادفية (Stochastic Processes). ويعد أسلوب سلاسل مـاركوف (Markov Chains) أحد التطبيقات العملية التصانسة التطادفية

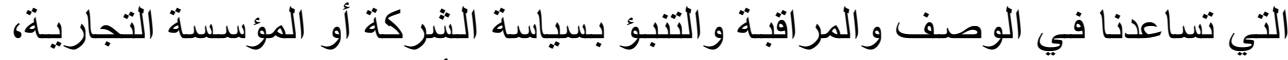

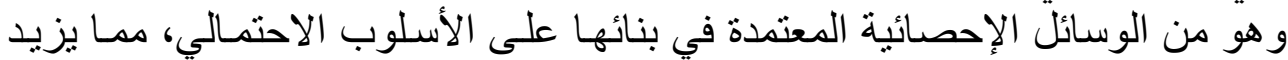

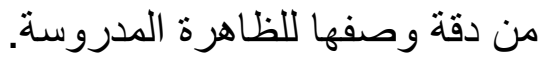

يهدف البحث إلى بيان الجانب التطبيقي و العملي للرياضيات الحديثة و المتمثلة

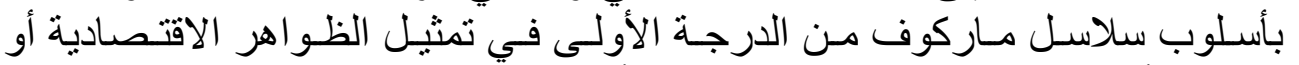

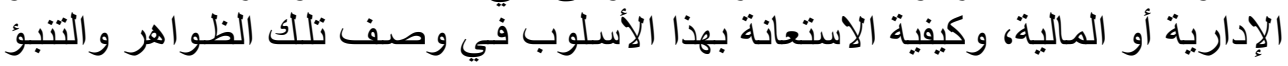

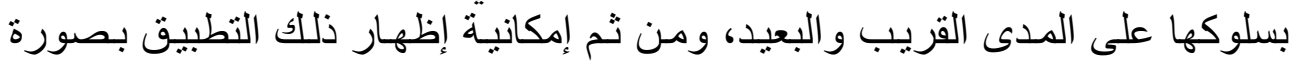

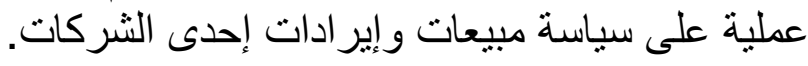

سنتناول في هذا المبحث بعض التعاريف الأساسية و الصيغ الرياضية الخاصـة ا ـ الجاتب النظري

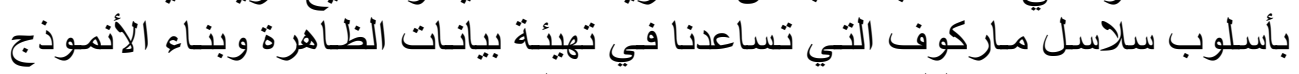

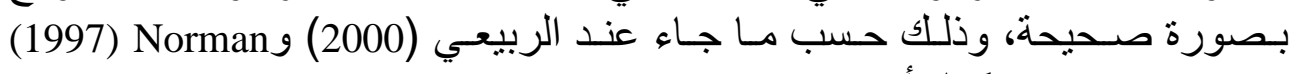
و Marius (1980) وكما يأني: 
1- 1 العملية التصادفية (Stochastic Processes)

يقال لأية ظاهرة حقيقية تجرى في حيز معين كالتئ مالزمن مثلاً بأنها عملية تصادفية

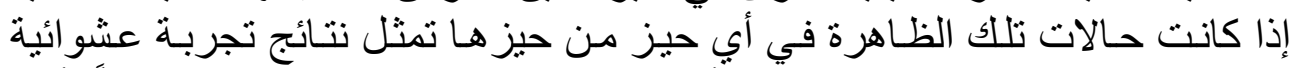

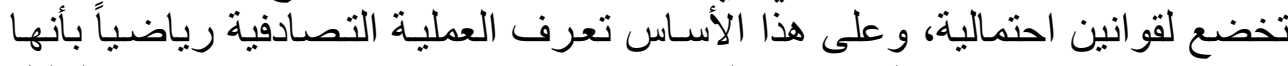

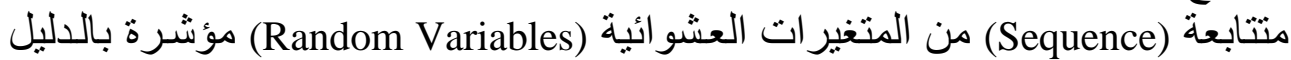

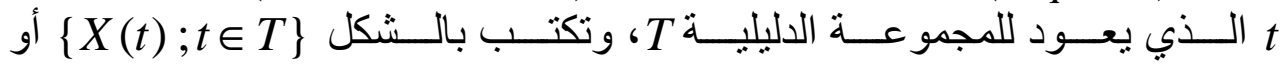
بالثكل $\{X(t)$

1. هنالك صفتان تشترك بهما أي عملية تصادفية هما فضاء الحفاء الحالة)(State Space) وفضاء المعلمة (Parameter Space).

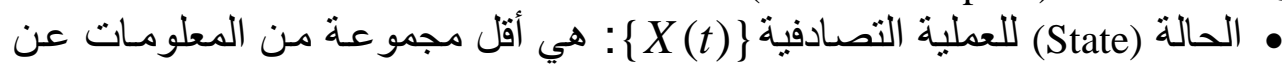
الظاهرة في الحاضر أو الماضي بحيث يمكن من خلالها وصف السلوك المستقبلي للنظام. فضاء الحالة (State Space): هي المجمو عـة التي تصم جميع الحسالات الممكنة

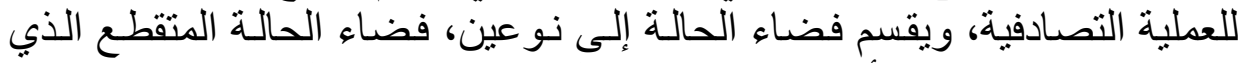

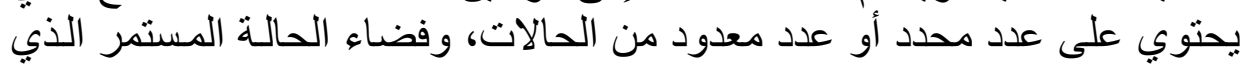
يحتوي على عدد غير محدد من الحالات.

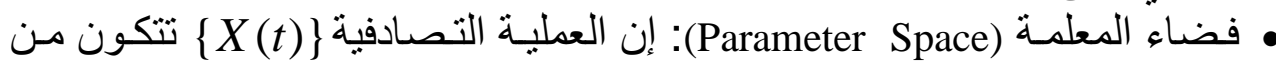

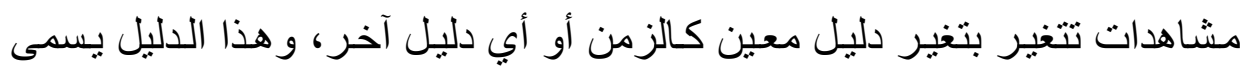

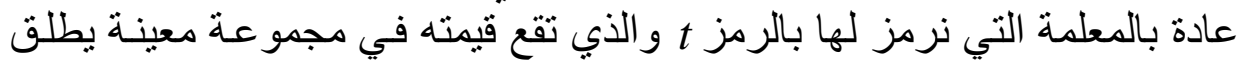

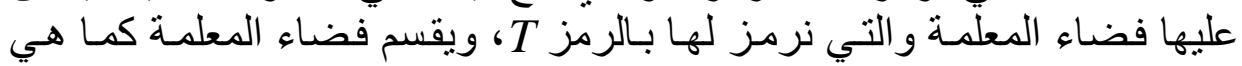

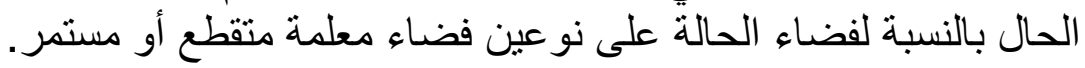

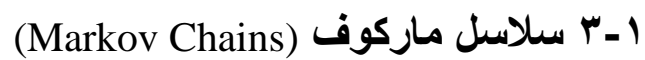

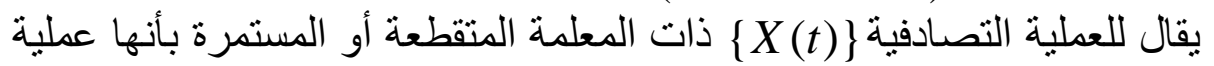
ماركوف إذا حققت الخاصية الآتية والتي تعرف بخاصية ماركوف.

$$
P\left(X_{t+1}=j \mid X_{t}=i, \mathrm{~L}, X_{0}=k\right)=P\left(X_{t+1}=j \mid X_{t}=i\right)=P_{i j}
$$

بمعنى أن سلسلة ماركوف هي حالة خاصة من العملية التصادفية عندما يعتمد

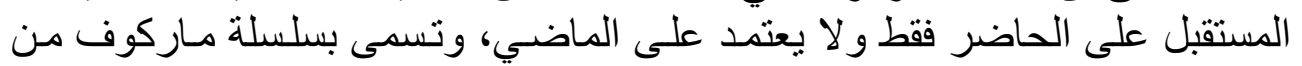

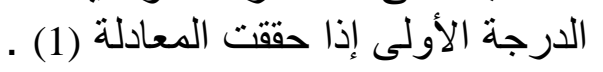




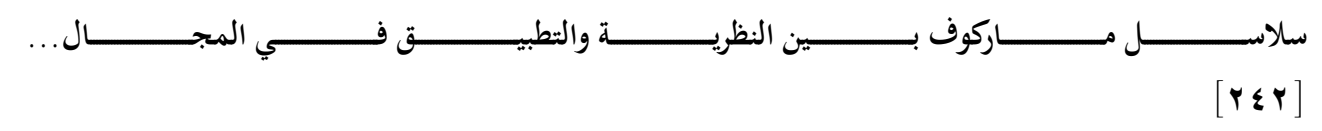

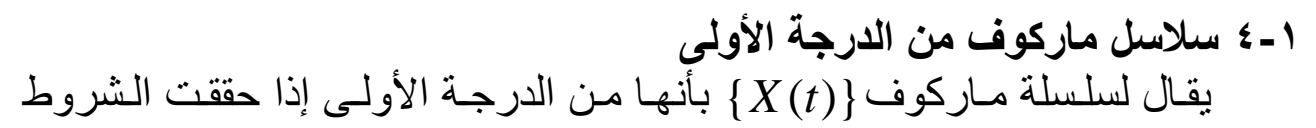

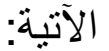
• الاحتمال نتائج محاولة معينة يعتمد في أكثر الأحيان على نتائج المحاولة التي • • إذا كان احتمال أبي محاولة ثابتاً بالنسبة للمحاو لات الأخرى من السلسلة الزمنية. إذا كان عدد النتائج المككن حدوثها محدوداً.

إن الاحتمالات الانتقالية (Transition Probability)

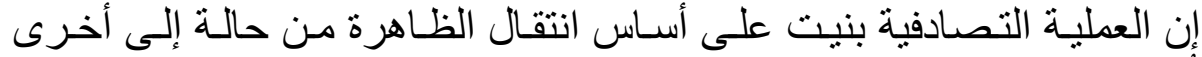

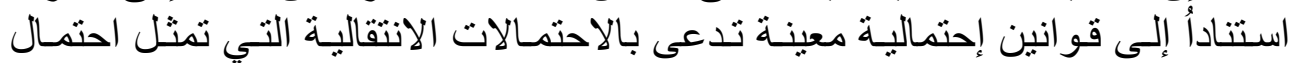
الانتقال خلال فترة زمنية معينة نرمز لها بالرمز الإن

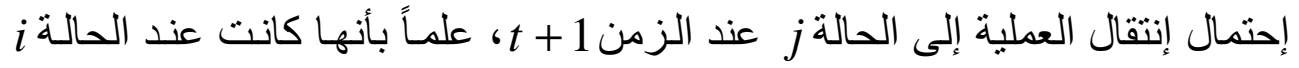

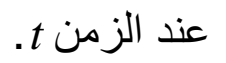

\section{r ـ وصف الظاهرة باستخدام سلاسل ماركوف وتقاير الاحتمالات الاتثقالية}

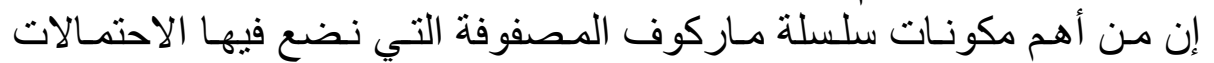

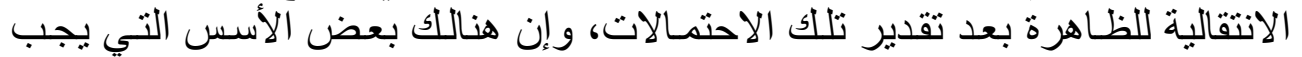

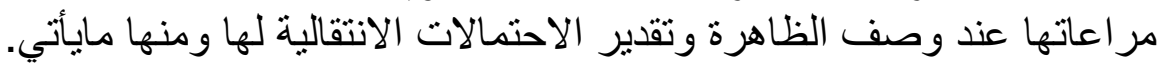

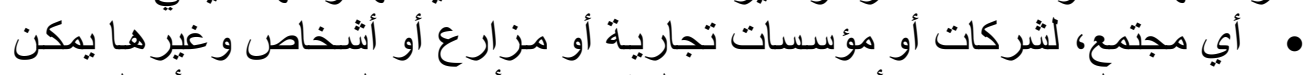

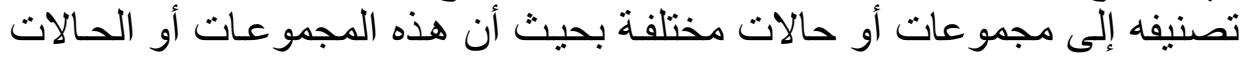
يجب أن لا تكون منتابكة فيما بينها. التنقل بين هذه المجمو عات أو الحالات عبر أي فترة زمنية يتم بشكل عشو ائي ثبات احتمالات الانتقال خلال فترة الحساب و التتبؤ.

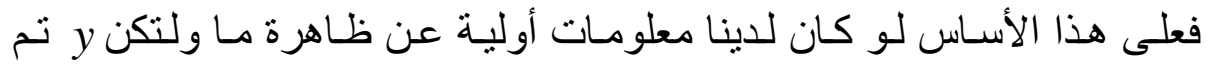

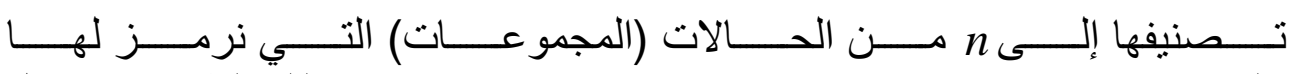

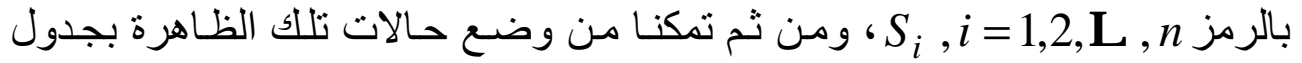

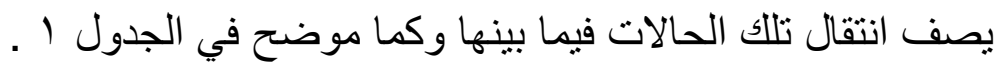




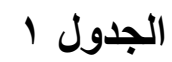

كيفية توزيع قيم الظاهرة y على

\begin{tabular}{|c|c|c|c|c|c|c|c|}
\hline الحالات & $\overline{S_{1}}$ & $S_{2}$ & 八 & $\overline{S_{j}}$ & ר & $S_{n}$ & المجموع عند الفترة t \\
\hline$S_{1}$ & $y_{11}$ & $y_{12}$ & 八 & $y_{1 j}$ & 八 & $y_{1 n}$ & $y_{1}$ \\
\hline$S_{2}$ & $y_{21}$ & $y_{22}$ & 八 & $y_{2 j}$ & 八 & $y_{2 n}$ & $y_{2}$ \\
\hline$M$ & $M$ & $M$ & 八 & $M$ & $\therefore$ & $\bar{M}$ & $\bar{M}$ \\
\hline$S_{i}$ & $y_{i 1}$ & $y_{i 2}$ & $\therefore$ & $y_{i j}$ & 八 & $y_{\text {in }}$ & $y_{i}$ \\
\hline$M$ & $M$ & $M$ & 八 & $\bar{M}$ & $\therefore$ & $M$ & $\bar{M}$ \\
\hline$S_{n}$ & $y_{n 1}$ & $y_{n 2}$ & 八 & $y_{n j}$ & 八 & $y_{n n}$ & $y_{n}$ \\
\hline 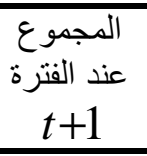 & $y_{\cdot 1}$ & $y_{\cdot 2}$ & N & $y_{\cdot j}$ & י & $y_{\cdot n}$ & $y=y_{t}=y_{t+1}$ \\
\hline
\end{tabular}

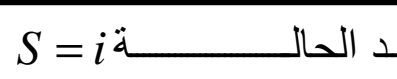

لظ

إذ إن قيم

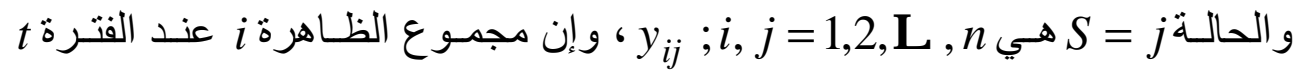
هـو n, ـ

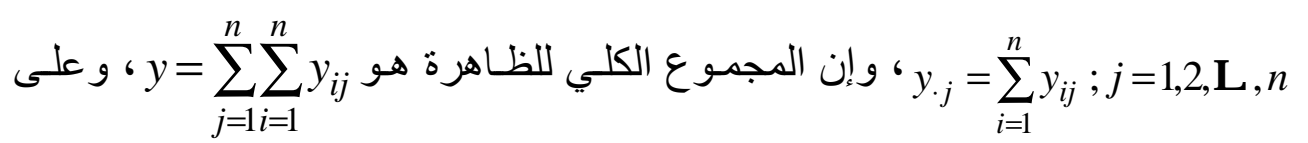

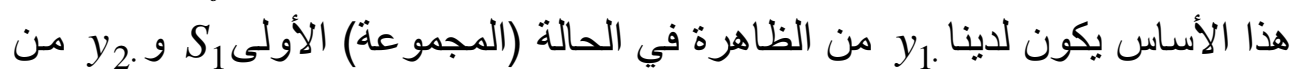

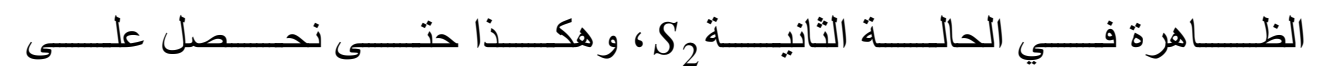

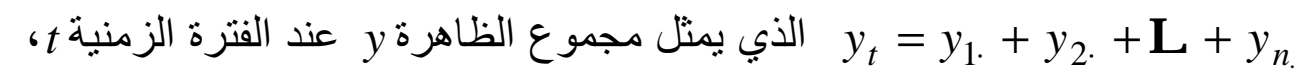
مقابل ذللك يوجد

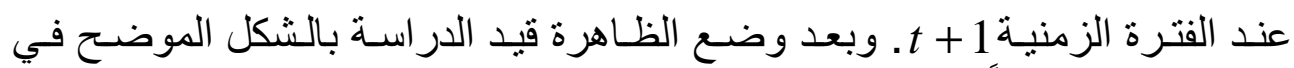

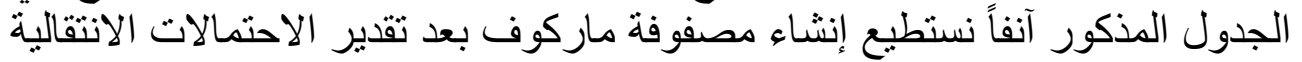

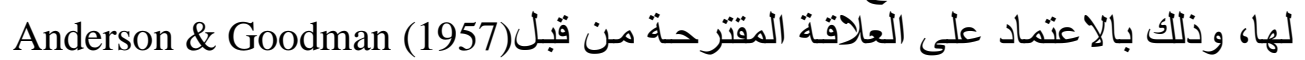
في حالة توفر فترة زمنية واحدة والمعرفة حسب المنية المعادلة الآتية: $P_{i j}=\frac{y_{i j}}{y_{i}} \quad ; i, j=1,2, \mathrm{~L}, n$ 


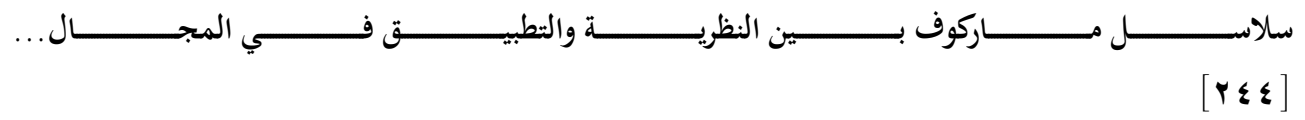

إن تفسير الاحتمال الناتج من المعادلة (2) يشابه الاحتمـال الذي تم تعريفه في

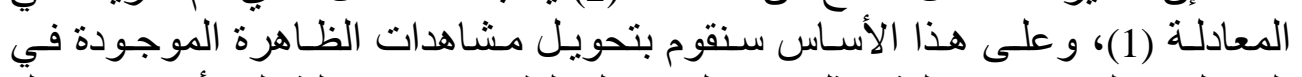

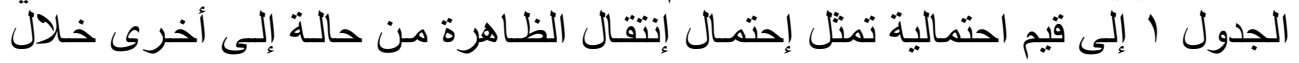

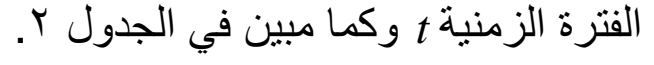

\begin{tabular}{|c|c|c|c|c|c|c|c|}
\hline \multicolumn{8}{|c|}{ الاحتمالات الانتقاليةّ المقدرة لقيم الظاهرة } \\
\hline الحالات & $S_{1}$ & $S_{2}$ & N & $S_{j}$ & N & $S_{n}$ & المجموع \\
\hline$S_{1}$ & $P_{11}$ & $P_{12}$ & N & $P_{1 j}$ & N & $P_{1 n}$ & 1 \\
\hline$S_{2}$ & $P_{21}$ & $P_{22}$ & י & $P_{2 j}$ & $\therefore$ & $P_{2 n}$ & 1 \\
\hline$M$ & $M$ & $M$ & N & $M$ & י & $M$ & 1 \\
\hline$S_{i}$ & $P_{i 1}$ & $P_{i 2}$ & N & $P_{i j}$ & N & $P_{i n}$ & 1 \\
\hline$M$ & $M$ & $M$ & N & $M$ & N & $M$ & 1 \\
\hline$S_{n}$ & $P_{n 1}$ & $P_{n 2}$ & N & $P_{n j}$ & N & $P_{n n}$ & 1 \\
\hline
\end{tabular}

لقد جرت العادة على وضع الاحتمالات الانتقالية للظاهرة على شكل مصفوفة

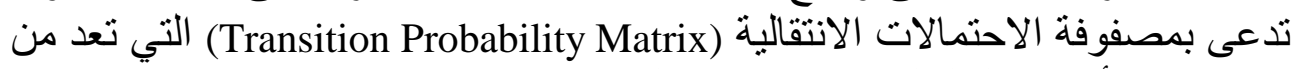

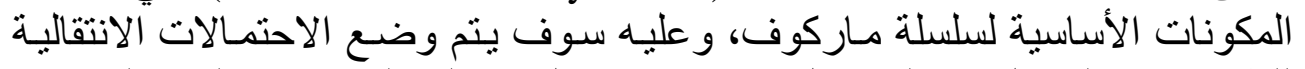

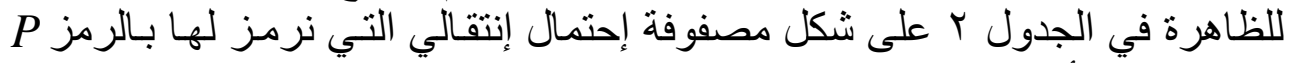

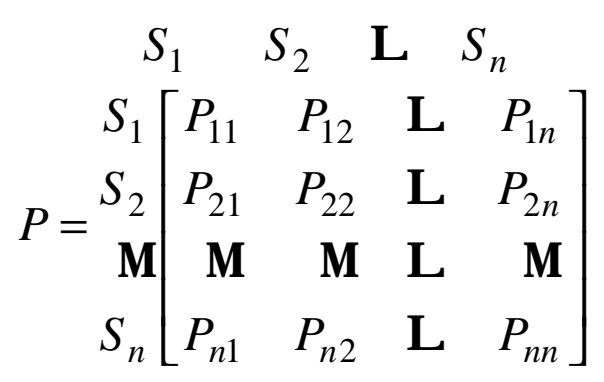
وكما موضتح أدناه.

ومن مميز ات هذه المصفوفة ما يأتي:

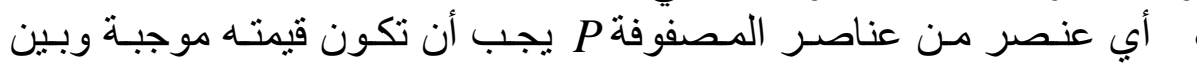
إن مجموع أبي صـف مـن صـفوف المـصفوفة P يجـب إن يساوي الواحد الصحيح. إن المصفوفة P من الدرجة $n$ × أب يجب أن تكون مصفوفة مربعة. 
r. كيفية استخدام أنموذج سلسلة ماركوف للتنبؤ بالظاهرة قيد الدراسة

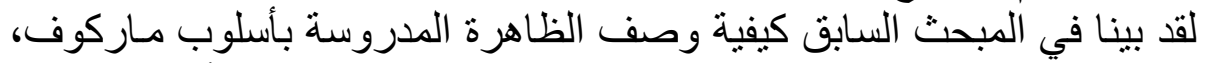

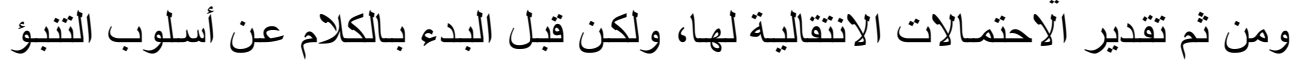

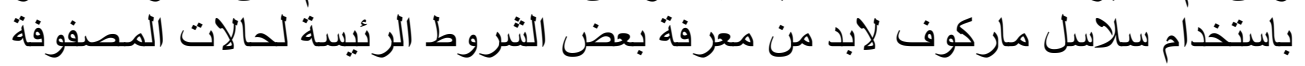

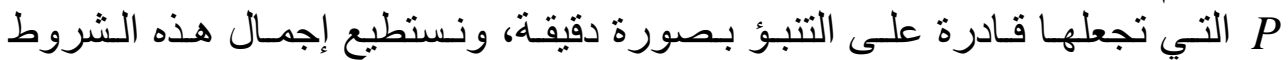

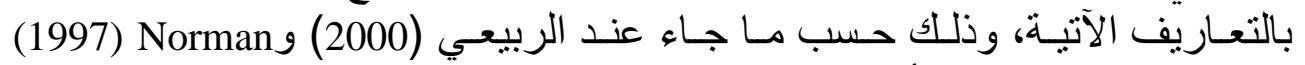
و Marius (1980) وكما يأني:

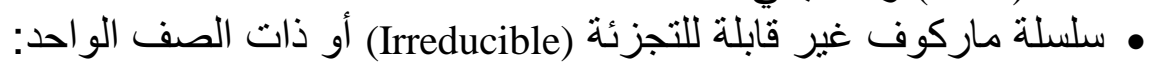

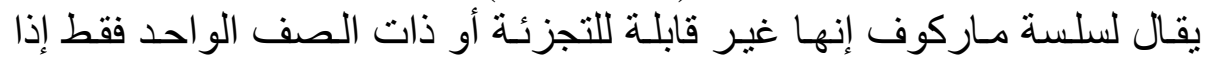

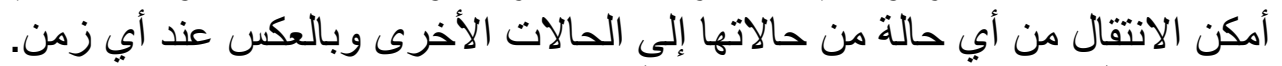
• سلسلة ماركوف ذات ذات العودة الموجبة (Positive Recurrent)

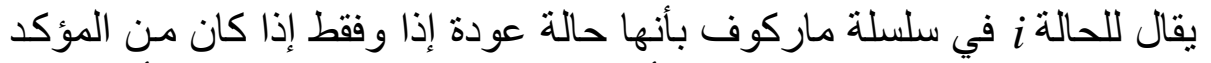

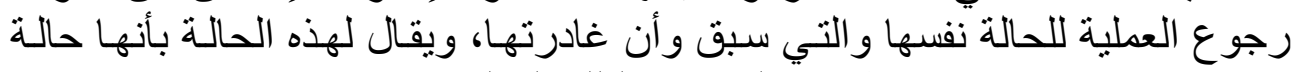

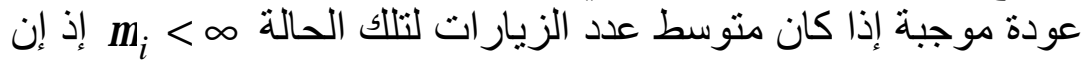
$\mu_{i}=\sum_{n=1}^{\infty} n f_{i i}^{n} \quad ; f_{i i}^{n}=P\left(y_{n}=i, y_{r} \neq i, r=0,1, \mathrm{~L}, n-1 \mid y_{0}=i\right) \mathrm{L}$

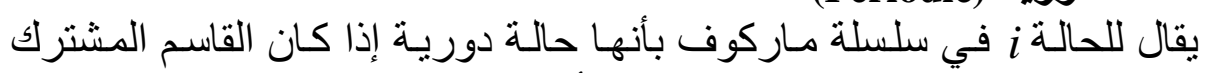

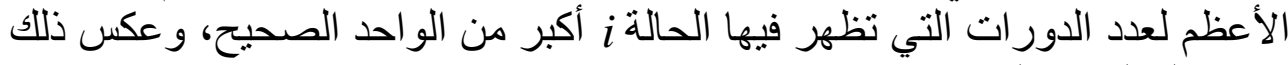
تسمى الحالة $i$ حالة غير دورية.

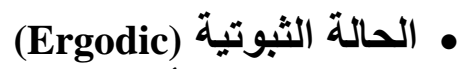

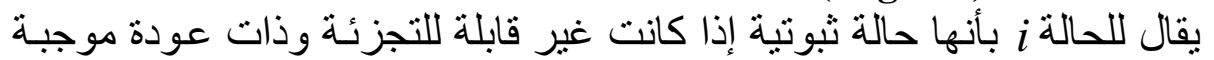
و غير دورية. إذا تو افرت هذه الشروط الأربعـة في سلسلة مساركوف نستطيع بعدها إيجـاد

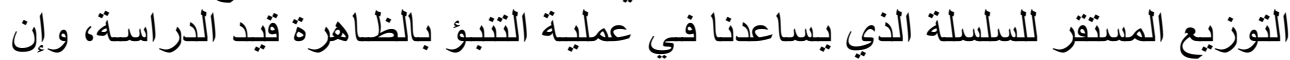

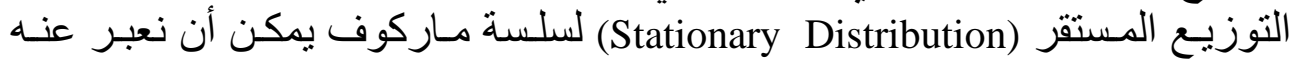

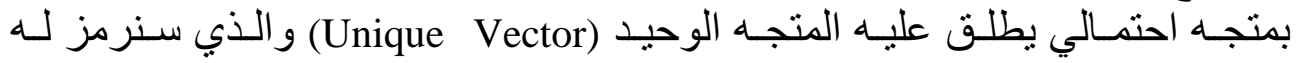

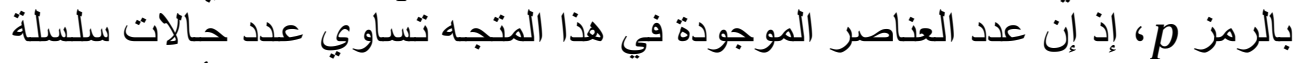

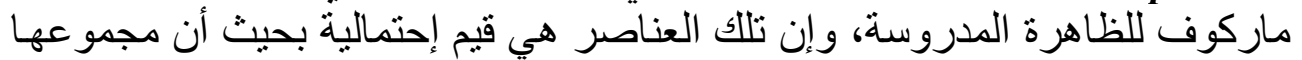

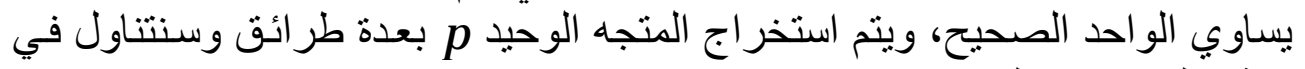
بحثنا طريقتين فقط. 


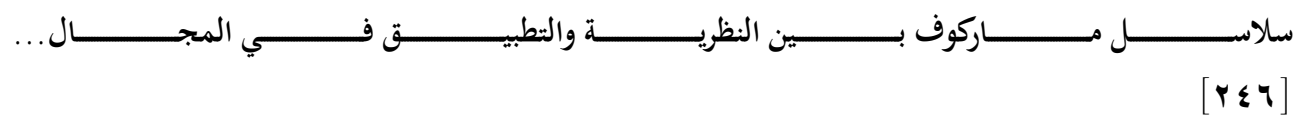

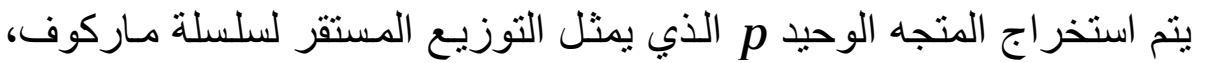

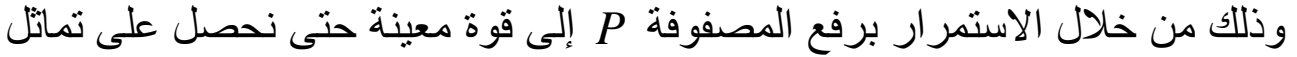

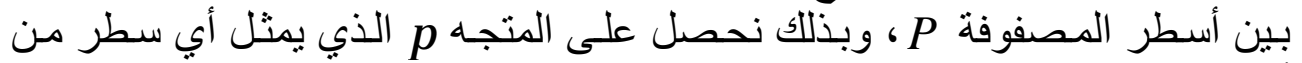

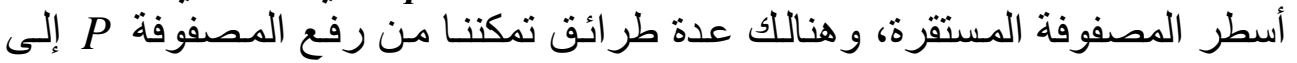
$P^{n}=P P^{n-1} \quad ; n \geq 1$

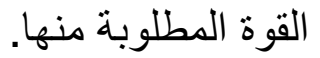

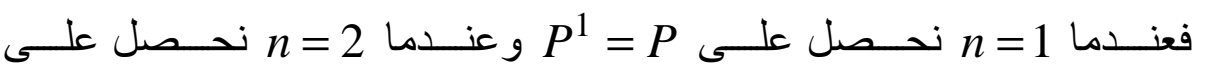
و هكذا إلى أي قوة. بحيث أن القوة التي ترفع لها المصفوفة تدل

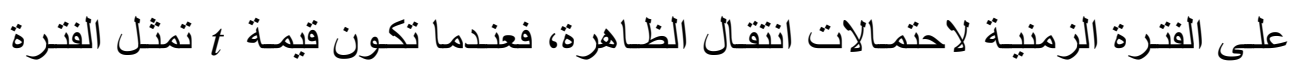

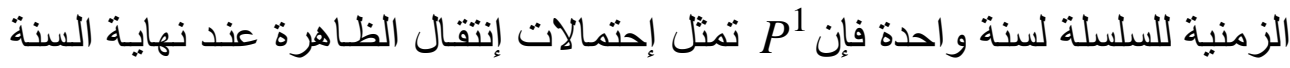

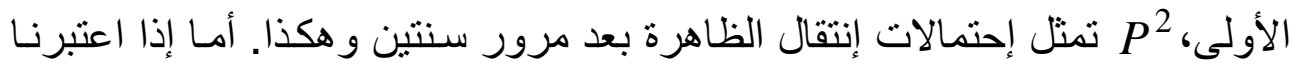

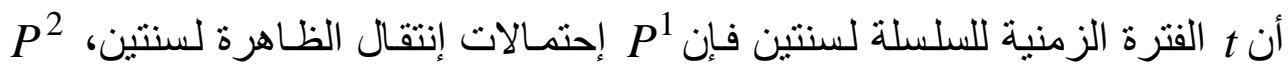

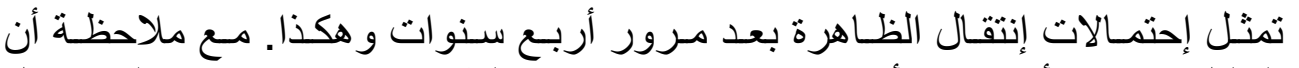

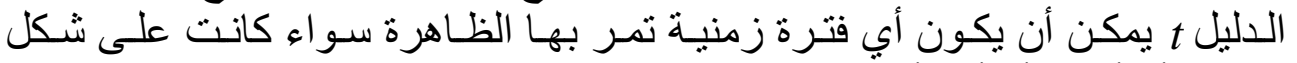
سنوات أو أثنهر أو أيام أو ساعات و وغيرة هال

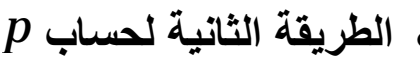

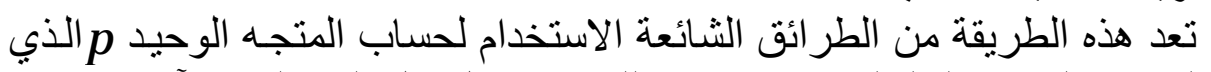
يمثل التوزيع المستقر لسلسلة ماركوف، وذللك من خلال حل المعادلتين الآتيتين. $\pi_{j}=\sum_{i=1}^{n} \pi_{j} P_{i j} \quad ; j \geq 1 \Rightarrow \pi P=\pi$

$\sum_{j=1}^{n} \pi_{j}=1$

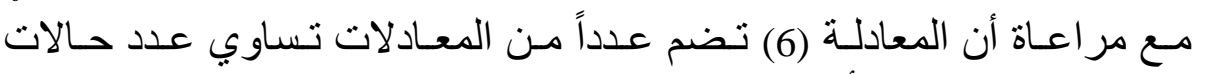

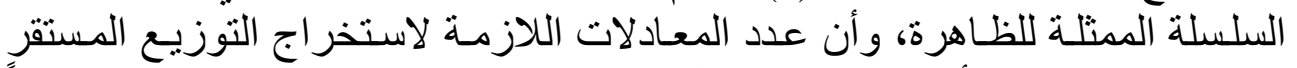

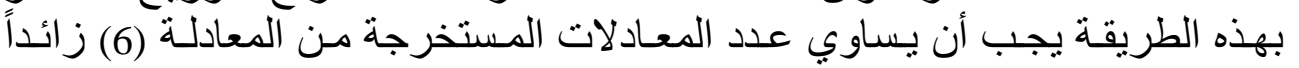

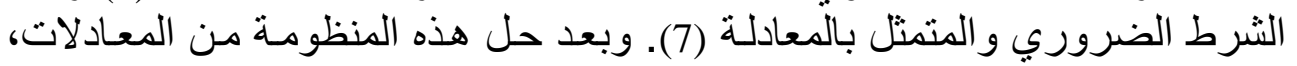

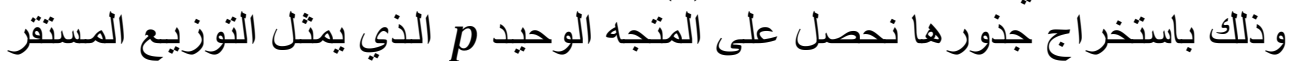

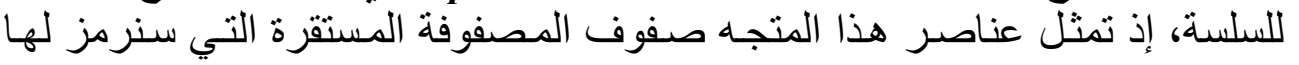

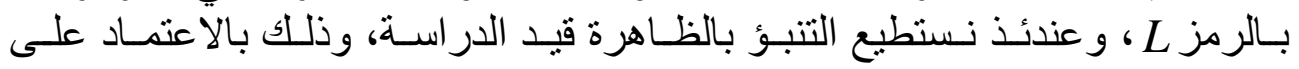

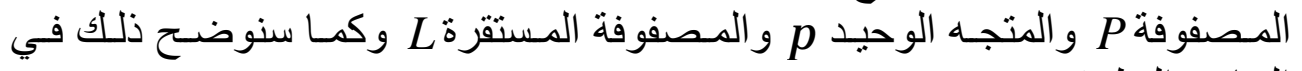
الجانب التطبيقي

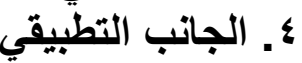




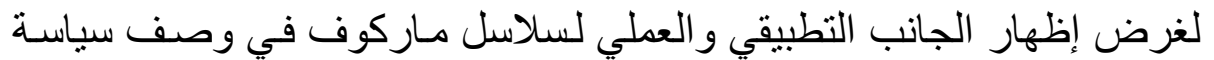

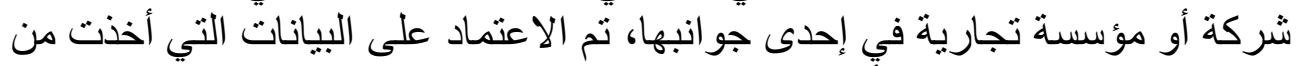

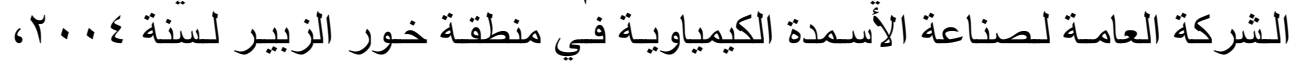

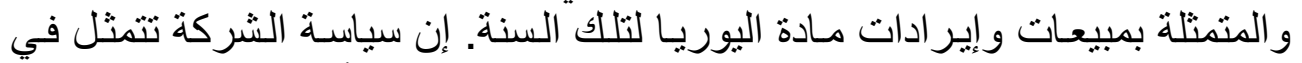

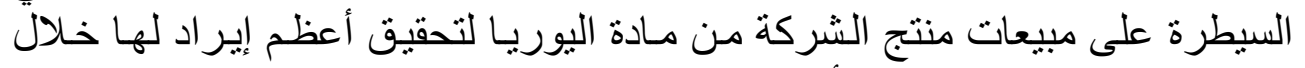

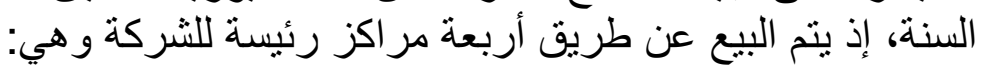

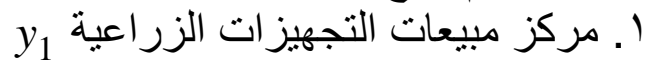
y

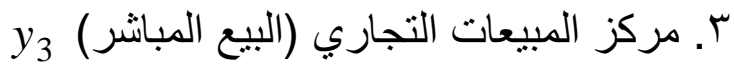

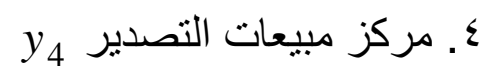

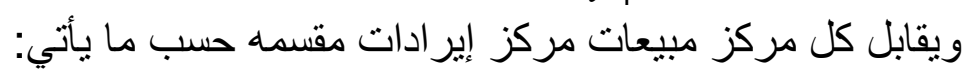

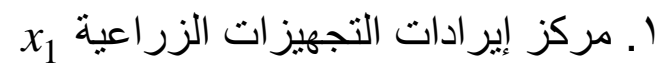

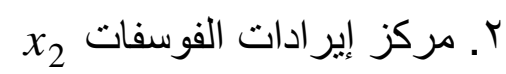

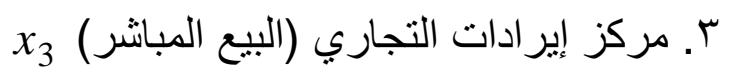

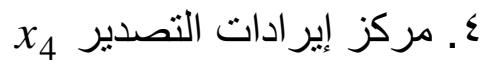

ـ ــ الهيئة البيانات لأنموذج ماركوف اليان

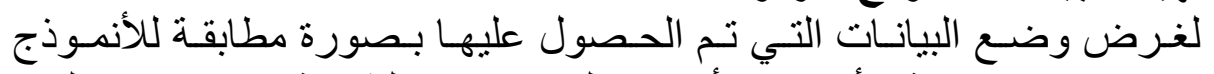

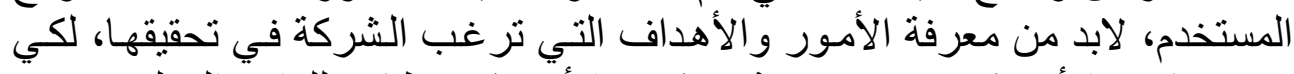

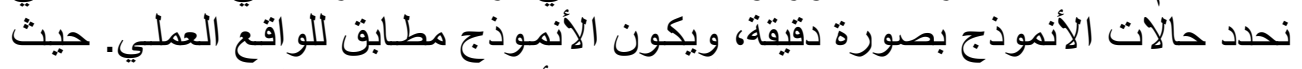

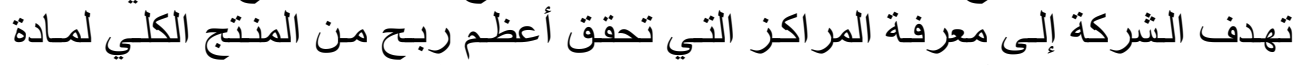

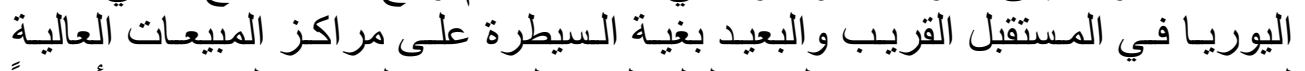

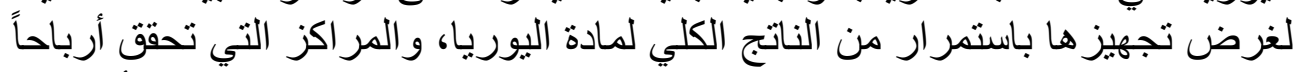

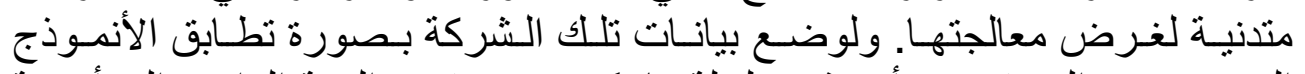

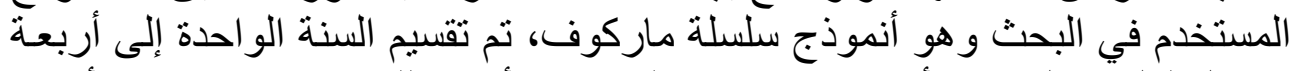

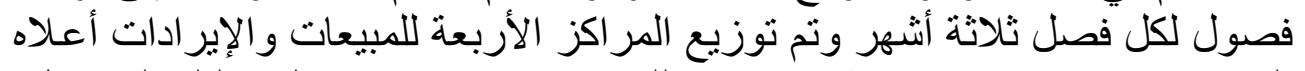

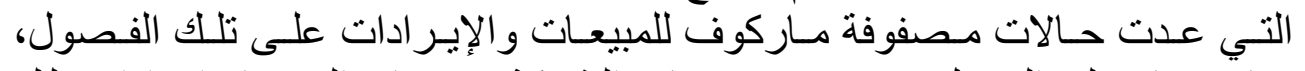

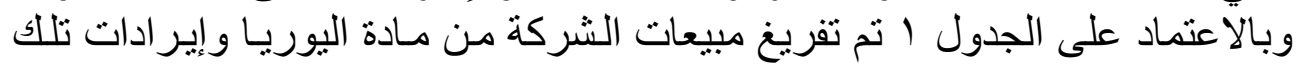

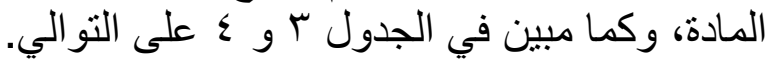




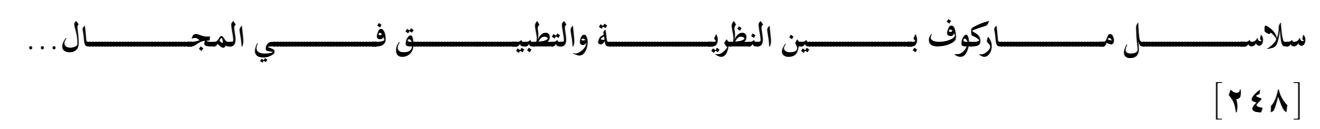

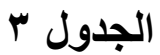

مبيعات مادة اليوريا بالطن موزعة على الحالات (المراكز) الأربعة

\begin{tabular}{|c|c|c|c|c|c|}
\hline الحالات & $y_{1}$ & $y_{2}$ & $y_{3}$ & $y_{4}$ & المجموع الفترة \\
\hline$y_{1}$ & 65064 & 16432.3 & 45622.93 & 46711.17 & 173830.4 \\
\hline$y_{2}$ & 50278 & 10350.9 & 17944 & 78048 & 156620.9 \\
\hline$y_{3}$ & 37412 & 12286.3 & 20950.1 & 37194.61 & 107843 \\
\hline$y_{4}$ & 63886 & 18541.7 & 12440.25 & 27855.19 & 122723.1 \\
\hline عند الفترة & 216640 & 57611.2 & 96957.28 & 189809 & 561017.4 \\
\hline
\end{tabular}

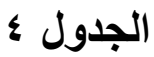

إيرادات مادة اليوريا بالاينار موزعة على الحالات (المراكز) الأربعة

\begin{tabular}{|c|c|c|c|c|c|}
\hline الحالات & $x_{1}$ & $x_{2}$ & $x_{3}$ & $x_{4}$ & لمجموع عند \\
\hline$x_{1}$ & $2.41 \mathrm{E}+09$ & $2.46 \mathrm{E}+08$ & $1.46 \mathrm{E}+08$ & $2.27 \mathrm{E}+09$ & $5.07 \mathrm{E}+09$ \\
\hline$x_{2}$ & $1.38 \mathrm{E}+09$ & $1.85 \mathrm{E}+08$ & $1.14 \mathrm{E}+09$ & $1.57 \mathrm{E}+09$ & $4.28 \mathrm{E}+09$ \\
\hline$x_{3}$ & $1.8 \mathrm{E}+09$ & $1.55 \mathrm{E}+08$ & $2.4 \mathrm{E}+09$ & $1.17 \mathrm{E}+09$ & $5.53 \mathrm{E}+09$ \\
\hline$x_{4}$ & $2.36 \mathrm{E}+09$ & $2.79 \mathrm{E}+08$ & $8.57 \mathrm{E}+08$ & $8.46 \mathrm{E}+08$ & $4.35 \mathrm{E}+09$ \\
\hline $\begin{array}{c}\text { المجمو الفترة } \\
t+1 \\
t+1\end{array}$ & $7.96 \mathrm{E}+09$ & $8.66 \mathrm{E}+08$ & $4.55 \mathrm{E}+09$ & $5.85 \mathrm{E}+09$ & $1.92 \mathrm{E}+10$ \\
\hline
\end{tabular}

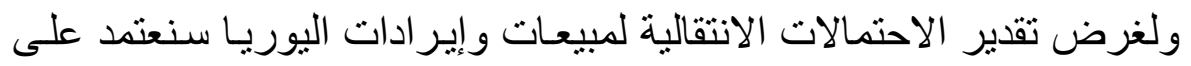

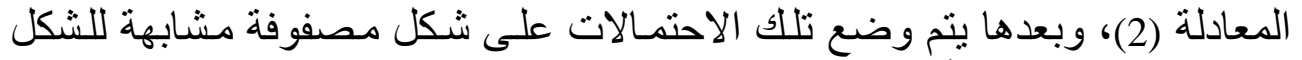

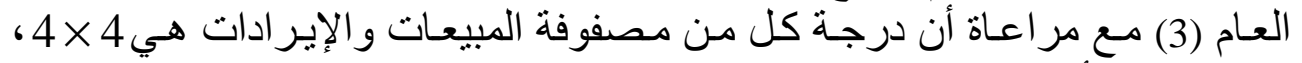

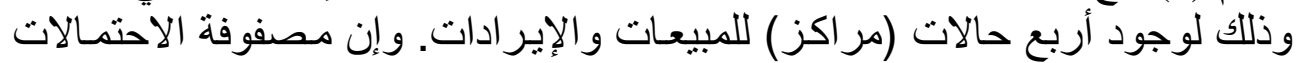
الانتقالية للمبيعات سنرمز لها بالرمز

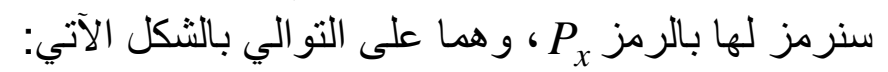

$\begin{array}{llll}y_{1} & y_{2} & y_{3} & y_{4}\end{array}$ 


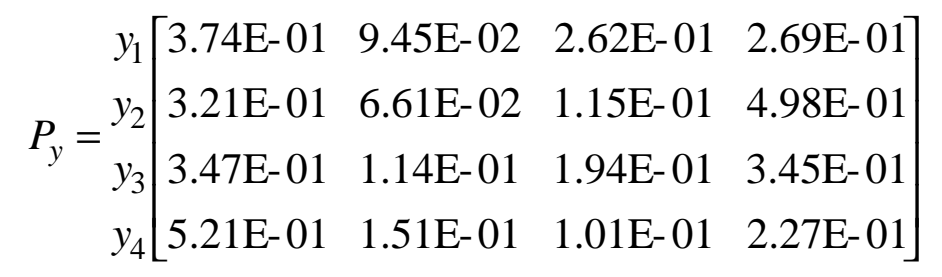

$$
\begin{aligned}
& P_{x}=\begin{array}{l}
x_{1} x_{2} \\
x_{3} \\
x_{3} \\
x_{4}
\end{array}\left[\begin{array}{lllll}
4.75 \mathrm{E}-01 & 4.86 \mathrm{E}-02 & 2.89 \mathrm{E}-02 & 4.47 \mathrm{E}-01 \\
3.23 \mathrm{E}-01 & 4.32 \mathrm{E}-02 & 2.67 \mathrm{E}-01 & 3.67 \mathrm{E}-01 \\
3.26 \mathrm{E}-01 & 2.81 \mathrm{E}-02 & 4.34 \mathrm{E}-01 & 2.12 \mathrm{E}-01 \\
5.44 \mathrm{E}-01 & 6.43 \mathrm{E}-02 & 1.97 \mathrm{E}-01 & 1.95 \mathrm{E}-01
\end{array}\right]
\end{aligned}
$$

يمكن تفسير القيم الواردة في المصفوفة P أو $P_{y}$ كما يأتي:

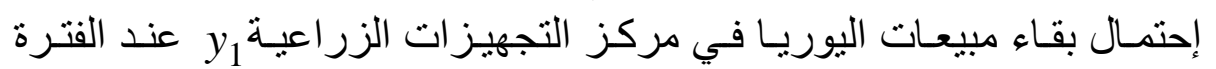

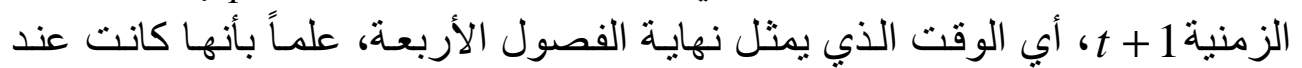

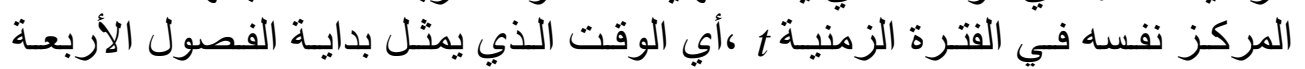

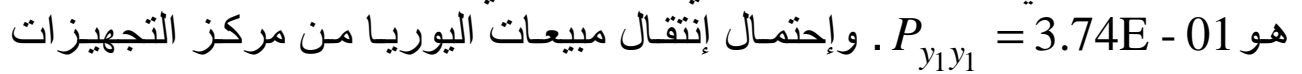

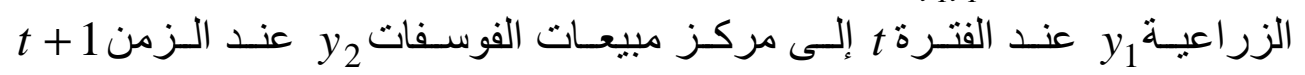

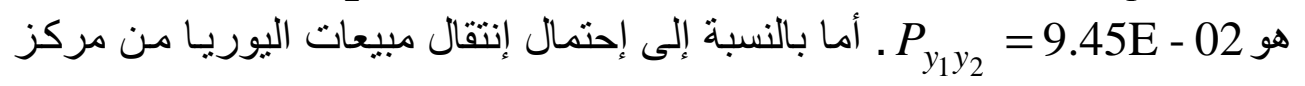
المبيعات التجارية $y_{3}$ عند الزمن t إلى مركز مبيعات التصدير

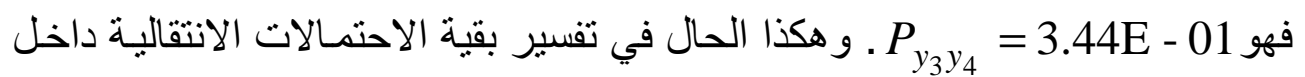
مصفوفة المبيعات P أو الاحتمالات الانتقالية لمصفوفة الإير ادات

\section{ع - التوزيع المستقر لمصفوفة المبيعات P}

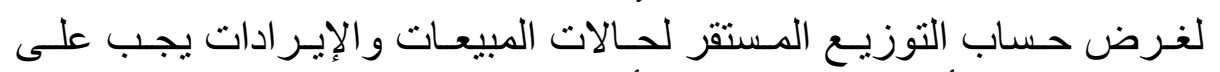
المصفوفتين

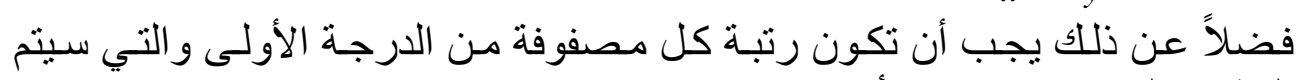

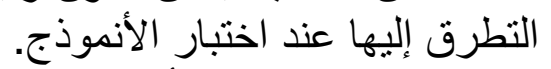
بالنسبة للثرط الأول نلاحظ أن كلا المصفوفنتين

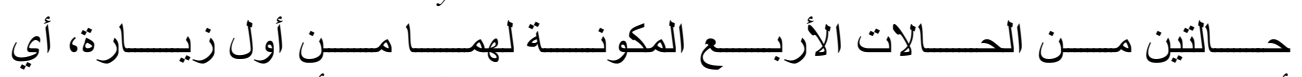
أن قابل قابلتين للتجزئة وكل منهما ينتمي إلى صف ر و احد. 


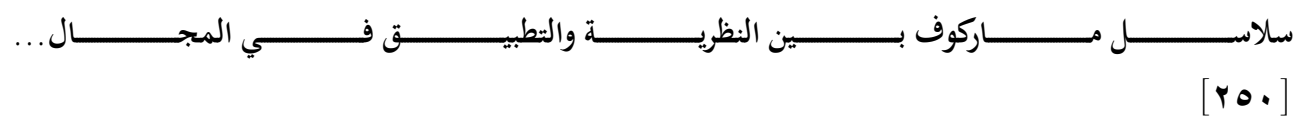

إن حالات المصفوفة P الأربع جميعها تكون ذات عودة موجبة، وذللك لأن متوسط عدد الزيار ات

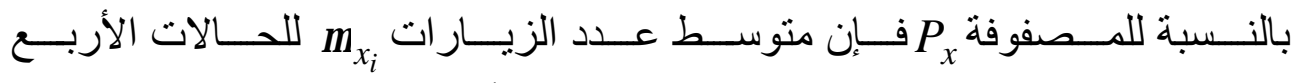

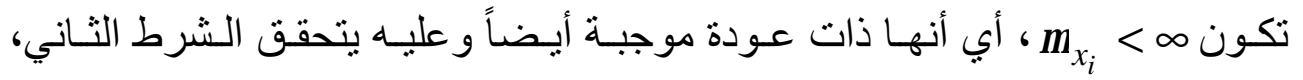

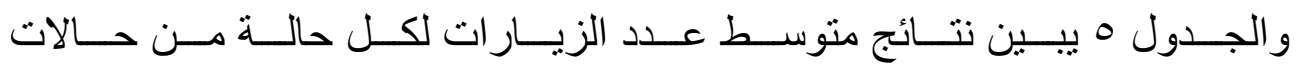
المصفوفة

\begin{tabular}{|c|c|c|c|c|}
\hline \multicolumn{5}{|c|}{ متوسط عدد الزيارات لحالات المبيعات $\mu_{x_{i}}$ ولحالات الإيرادات } \\
\hline الحالة $i$ & 1 & 2 & 3 & 4 \\
\hline$\mu_{y_{i}}$ & 2.4595 & 8.952 & 5.3886 & 3.3768 \\
\hline$\mu_{x_{i}}$ & 2.1463 & 19.9433 & 6.2414 & 3.0890 \\
\hline
\end{tabular}

بالنسبة للثرط الثالث يلاحظ أن حالات كلا المصفوفتنين

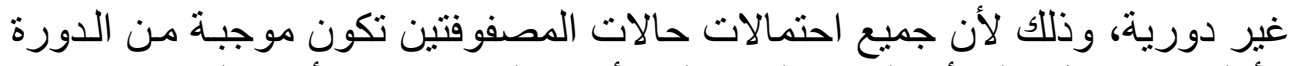

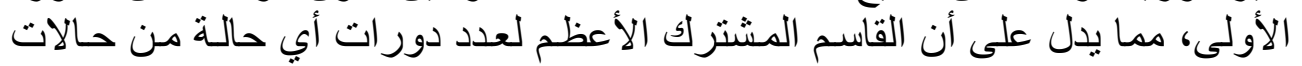
المصفوفتين هو الواحد الصحيح. بتحقق الشروط الثلاثة المذكورة آنفاً لكلا المصفوفنتين المصفو فتنين تكون ثابتة.

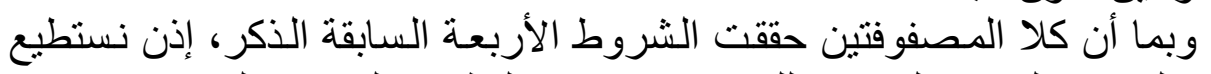

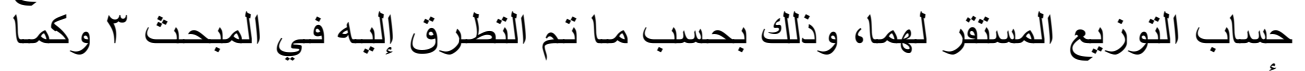

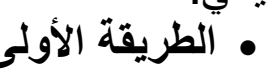

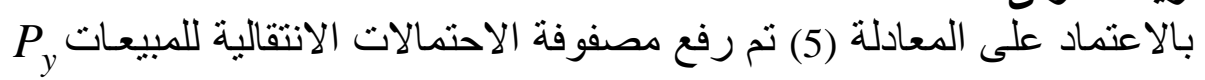

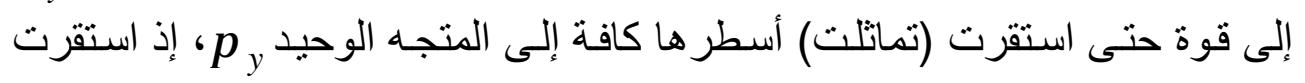

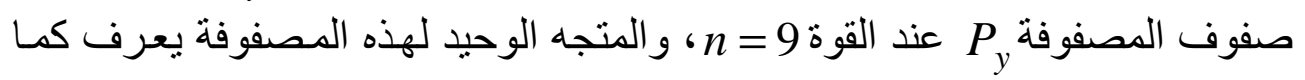
$\pi_{y}=\left[\begin{array}{llll}0.4066 & 0.11179 & 0.18562 & 0.2961\end{array}\right] \quad$ L (10)

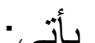
أما بالنسبة لمصفوفة الإير ادات ${ }^{\prime}$ فقد استقرت صفوفها عند القوة و المتجه الوحيد لهذه المصفوفة يعرف كما يأتي: 


$$
\begin{aligned}
& \pi_{x}=\left[\begin{array}{llll}
0.465912 & 0.050142 & 0.16022 & 0.323726
\end{array}\right] \quad \mathrm{L}(11) \\
& \text { م الطريقة الثانية }
\end{aligned}
$$

تم استخر اج التوزيع المستقر (المتجه الوحيد) لمصفوفة المبيعات والإيرادات، المكاته وذللك بالاعتماد على المعادلتين (6) و (7) على التو التي وكما مبين التين أدناه.

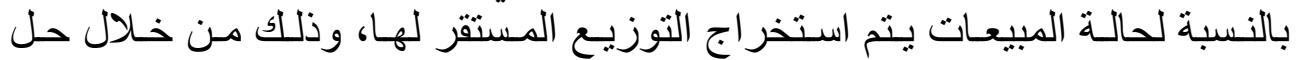

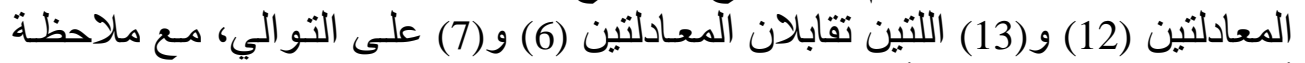

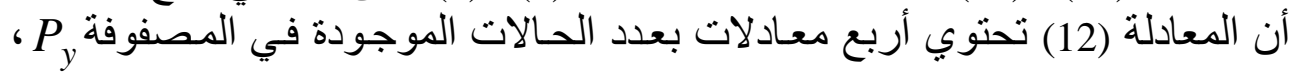

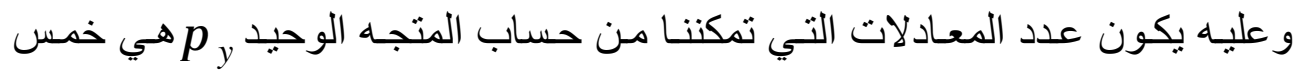

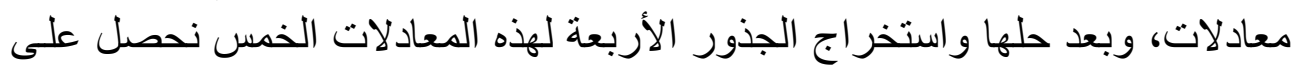

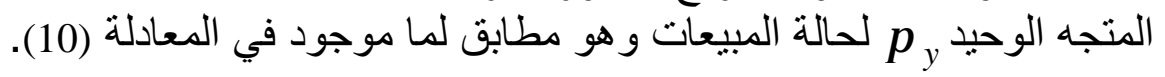
$\left[\pi_{y_{1}} \pi_{y_{2}} \pi_{y_{3}} \pi_{y_{4}}\right]\left[\begin{array}{llll}3.74 \mathrm{E}-01 & 9.45 \mathrm{E}-02 & 2.62 \mathrm{E}-01 & 2.69 \mathrm{E}-01 \\ 3.21 \mathrm{E}-01 & 6.61 \mathrm{E}-02 & 1.15 \mathrm{E}-01 & 4.98 \mathrm{E}-01 \\ 3.47 \mathrm{E}-01 & 1.14 \mathrm{E}-01 & 1.94 \mathrm{E}-01 & 3.45 \mathrm{E}-01 \\ 5.21 \mathrm{E}-01 & 1.51 \mathrm{E}-01 & 1.01 \mathrm{E}-01 & 2.27 \mathrm{E}-01\end{array}\right]=\left[\pi_{y_{1}} \pi_{y_{2}} \pi_{y_{3}} \pi_{y_{4}}\right] \mathrm{L}$ (12) $\pi_{y_{1}}+\pi_{y_{2}}+\pi_{y_{3}}+\pi_{y_{4}}=1 \quad \mathrm{~L}(13)$

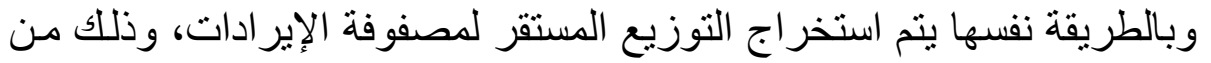

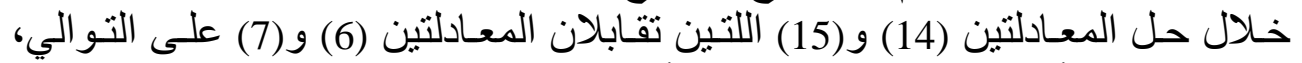

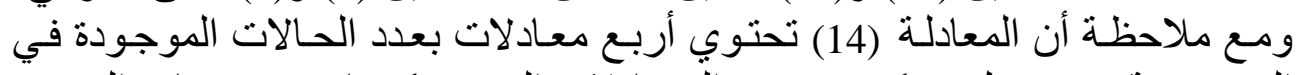

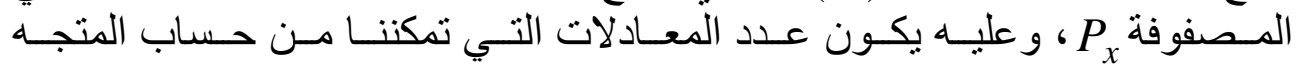

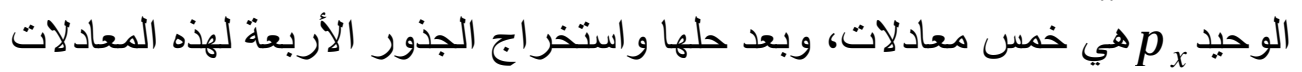

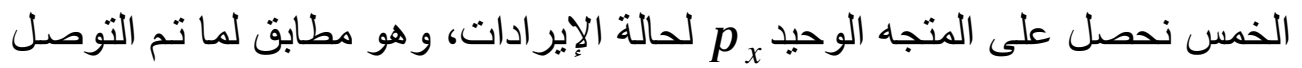
إليه في المعادلة (11) وكما يأتي: $\left[\pi_{x_{1}} \pi_{x_{2}} \pi_{x_{3}} \pi_{x_{4}}\right]\left[\begin{array}{llll}4.75 \mathrm{E}-01 & 4.86 \mathrm{E}-02 & 2.89 \mathrm{E}-02 & 4.47 \mathrm{E}-01 \\ 3.23 \mathrm{E}-01 & 4.32 \mathrm{E}-02 & 2.67 \mathrm{E}-01 & 3.67 \mathrm{E}-01 \\ 3.26 \mathrm{E}-01 & 2.81 \mathrm{E}-02 & 4.34 \mathrm{E}-01 & 2.12 \mathrm{E}-01 \\ 5.44 \mathrm{E}-01 & 6.43 \mathrm{E}-02 & 1.97 \mathrm{E}-01 & 1.95 \mathrm{E}-01\end{array}\right]=\left[\pi_{x_{1}} \pi_{x_{2}} \pi_{x_{3}} \pi_{x_{4}}\right] \mathrm{L}$ (14) $\pi_{x_{1}}+\pi_{x_{2}}+\pi_{x_{3}}+\pi_{x_{4}}=1 \quad \mathrm{~L}$ (15)

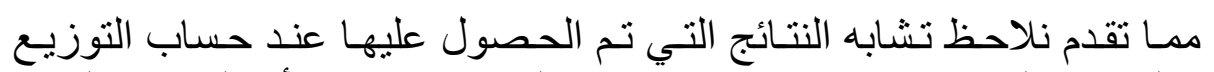

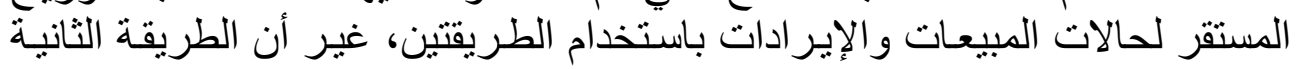
تعد الطريقة الامثل في الحساب. ت ع الإير 


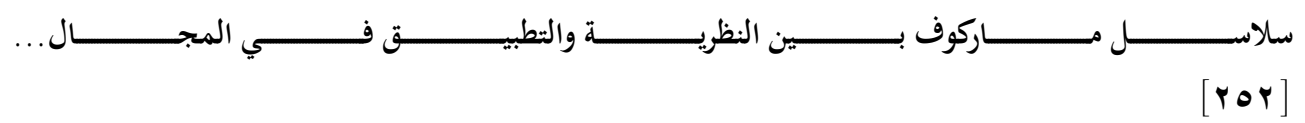

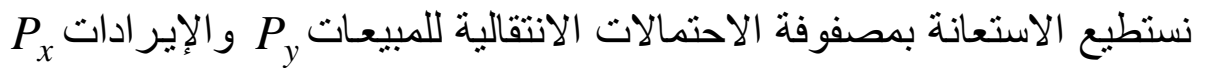

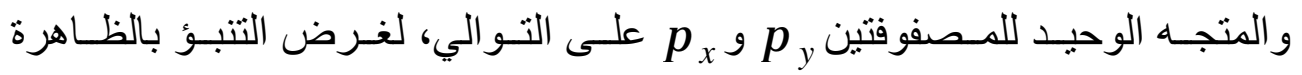

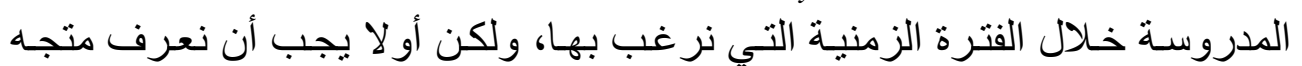
التشكيل الابتدائي للمبيعات

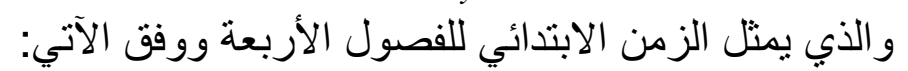
$C_{y t}=\left[\begin{array}{llll}173830.4 & 156620.9 & 107843 & 122723.1\end{array}\right]$

$C_{x t}=\left[\begin{array}{llll}5.07 \mathrm{E}+09 & 4.28 \mathrm{E}+09 \quad 5.53 \mathrm{E}+09 \quad 4.35 \mathrm{E}+09\end{array}\right]$

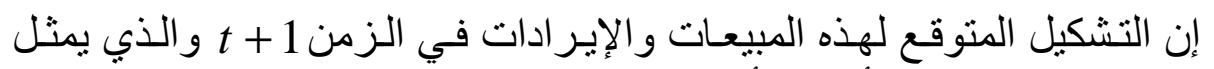

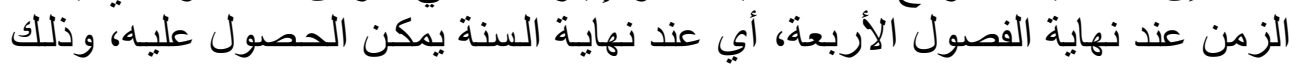

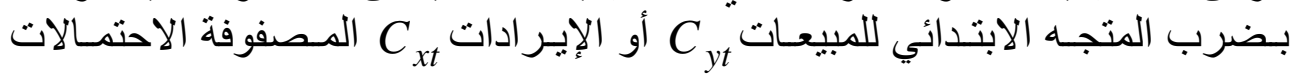

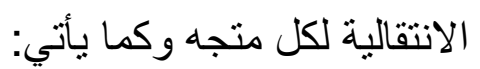

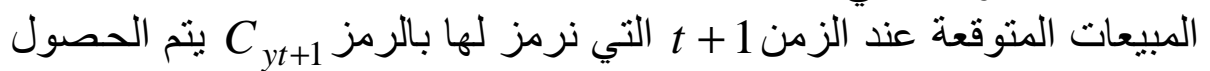

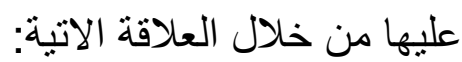
$C_{y t+1}=C_{y t} P_{y}=\left[\begin{array}{llll}216640 & 57611.2 & 96957.28 & 189809\end{array}\right]$

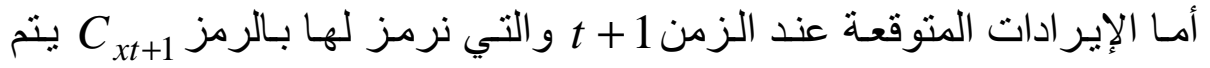
الحصول عليها من خلال العلاقة الآتية: $C_{x t+1}=C_{x t} P_{x}=\left[\begin{array}{llll}7.96 \mathrm{E}+09 & 8.66 \mathrm{E}+08 & 4.55 \mathrm{E}+09 & 5.85 \mathrm{E}+09\end{array}\right]$

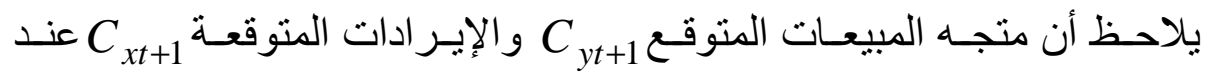

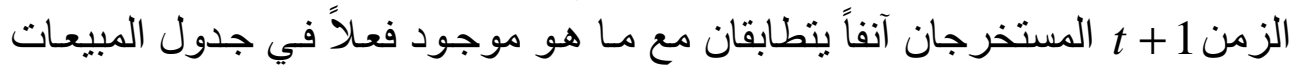

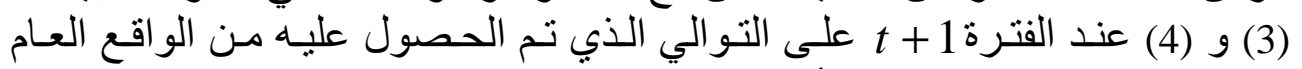

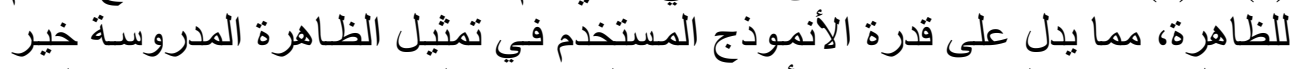

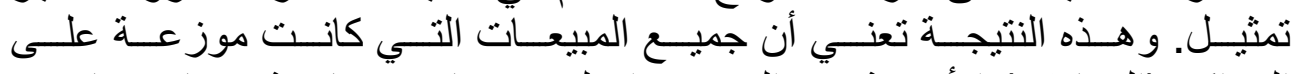

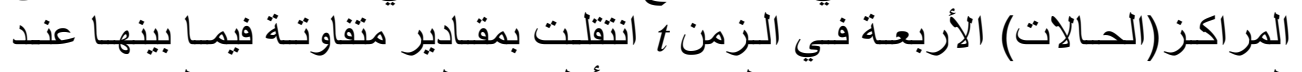

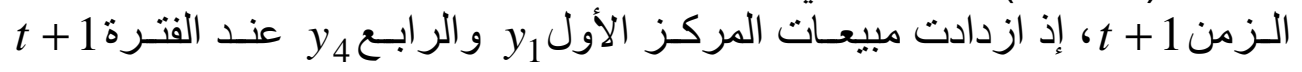

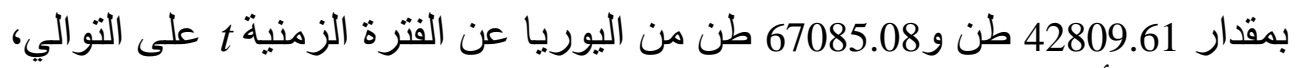

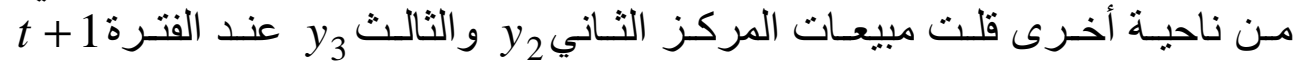

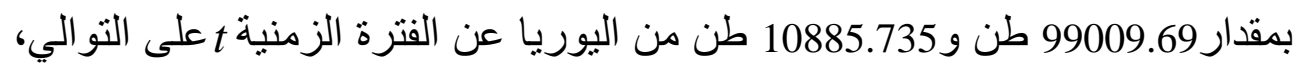

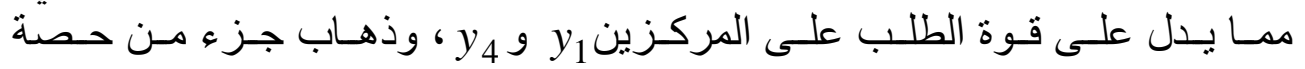
المركزين التوزيع بالنسبة للمركزين 


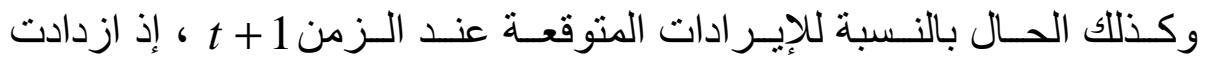

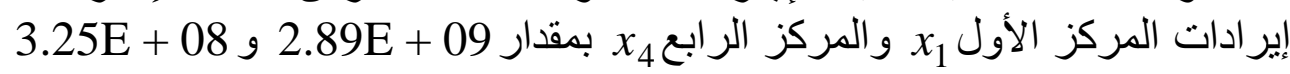

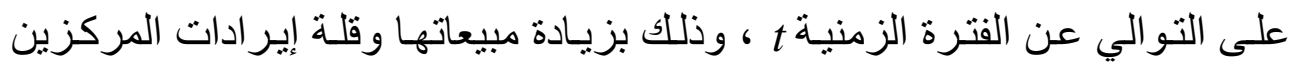

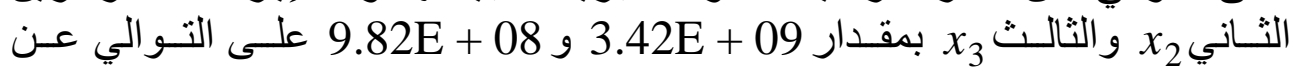
الفترة t و اللسبب المذكور نفسه.

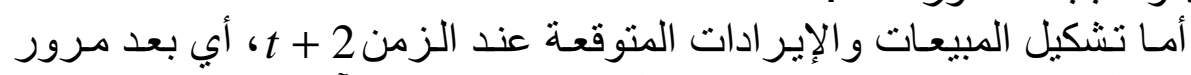

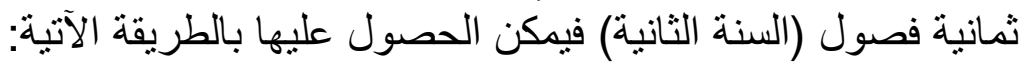

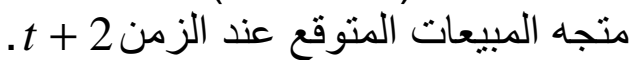
$C_{y t+2}=C_{y} P_{y}^{2}=\left[\begin{array}{llll}232026 & 64010 & 101535 & 163446\end{array}\right]$ $C_{x t+2}=C_{x} P_{x}^{2}=\left[\begin{array}{llll}8.73 \mathrm{E}+09 & 9.29 \mathrm{E}+08 & 3.59 \mathrm{E}+09 & 5.98 \mathrm{E}+09\end{array}\right]$ اذ يلاحظ على المتجهين المذكورين آنفاً بقاء مبيعات وإير ادات المرات المركزين الأول

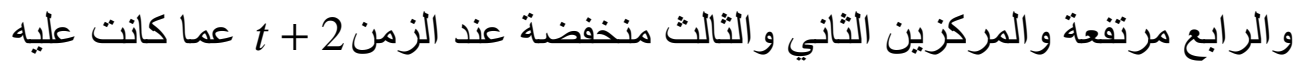
و عليه فإن المعادلة (20) و(21) أدناه تمكننا من التنبؤ بالمبيعات أو الإير ادات

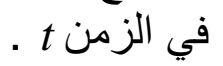

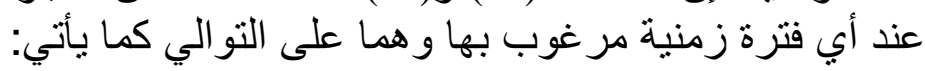
$C_{y t+n}=C_{y t} P_{y}^{n} \quad ; n=1,2, \mathrm{~L} \quad \mathrm{~L}(20)$ $C_{x t+n}=C_{x t} P_{x}^{n} \quad ; n=1,2, \mathrm{~L} \quad \mathrm{~L}(21)$

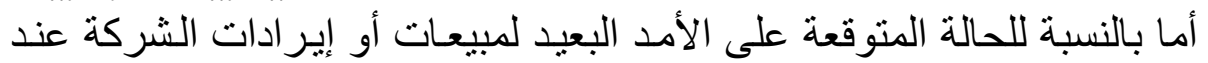

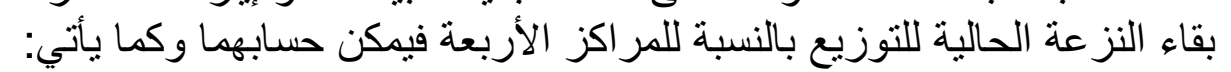
$C_{y \infty}=C_{y t} L_{y}=\left[\begin{array}{lllll}228110 & 62666 & 104125 & 166117\end{array}\right]$

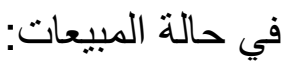
$C_{x \infty}=C_{x t} L_{x}=\left[\begin{array}{llll}8.96 \mathrm{E}+09 & 9.64 \mathrm{E}+08 & 3.08 \mathrm{E}+09 & \left.\begin{array}{c}\text { 6. } \\ \text { 6. }\end{array}\right]\end{array}\right.$ إذ إن المصفوفة

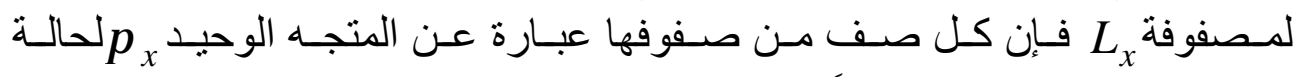

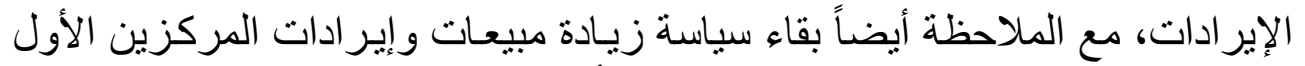

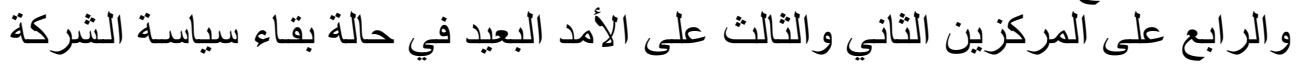

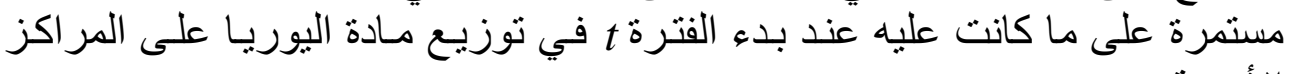

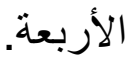




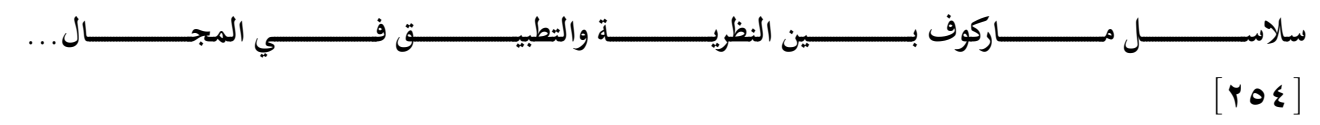

\section{هـ اختبار صلاحية الأنموذج للتقدير}

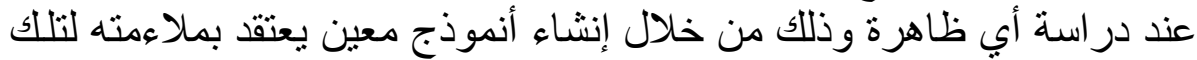

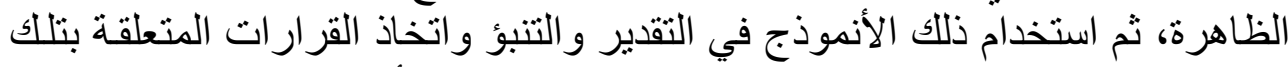

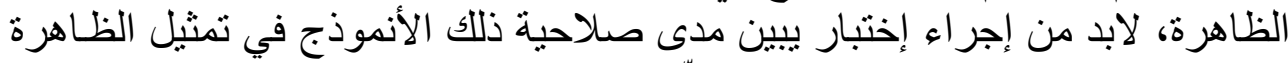

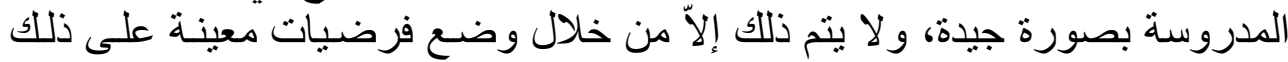

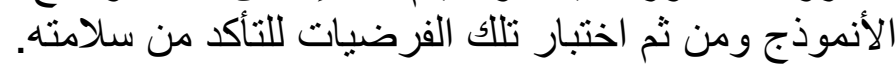

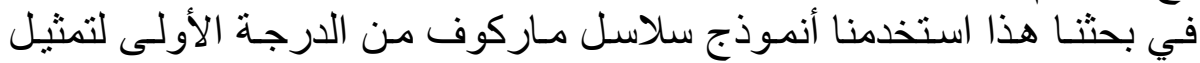

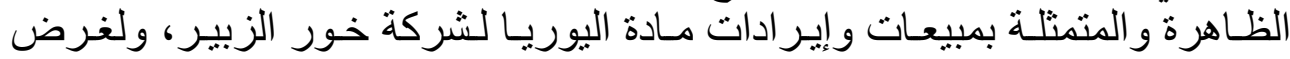

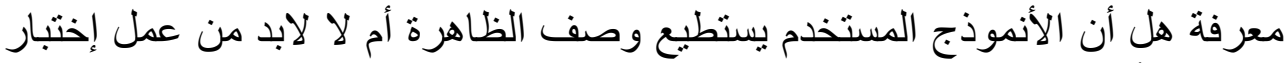

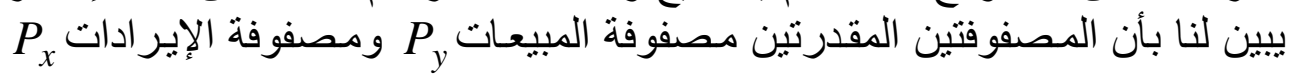

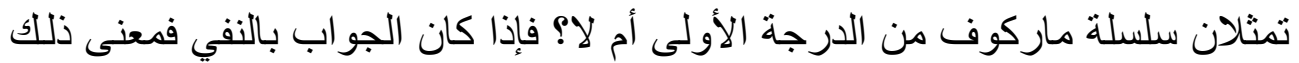

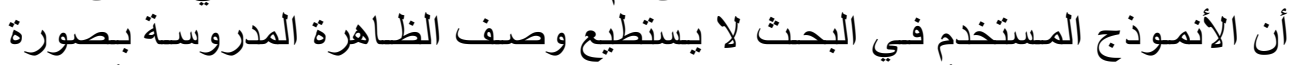

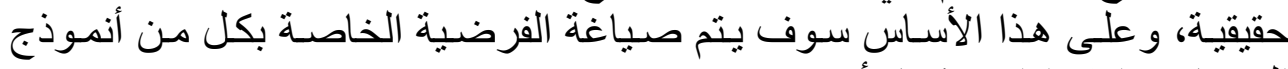
المبيعات و الإير ادات وكما يأتي: الإني

الفرضية الخاصة بأنموذج المبيعات

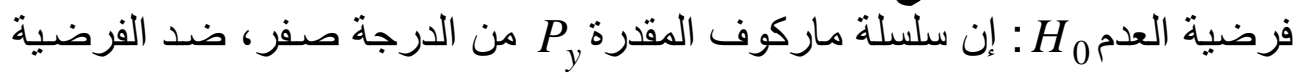

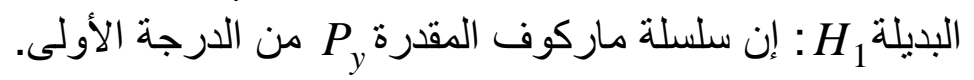

الفرضية الخاصة بأنموذج الإيرادات

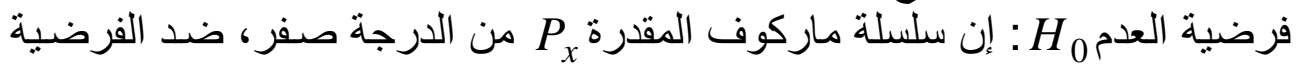

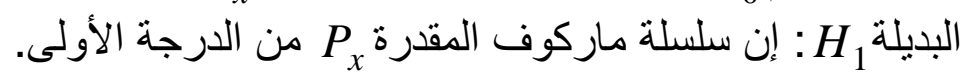

اذ سيتم إختبـار الفرضية الأولى باستخدام الاحصـاءه المعرفة عند الربيعي

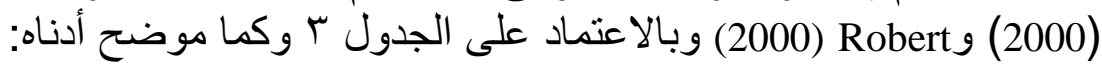

$\lambda_{y}=2 \sum_{j=1}^{m} \sum_{i=1}^{m} y_{i j} \log \frac{y_{i j}\left(\sum_{j=1}^{m} \sum_{i=1}^{m} y_{i j}\right)}{y_{i \cdot} y_{\cdot j}}$

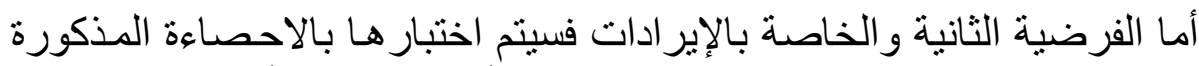

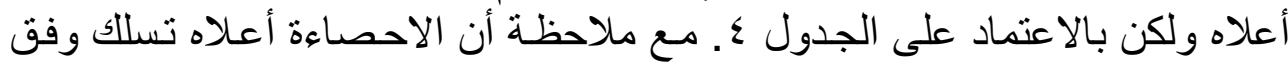

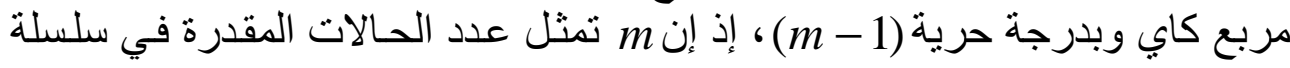

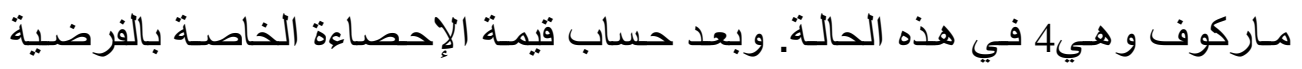




\section{[ أحمد [roo}

الأولى و التي تساوي تساوي 11 كائ

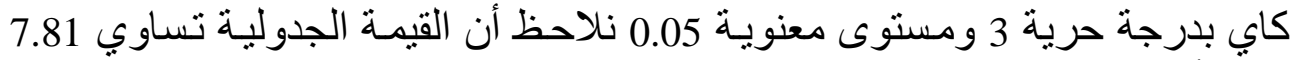

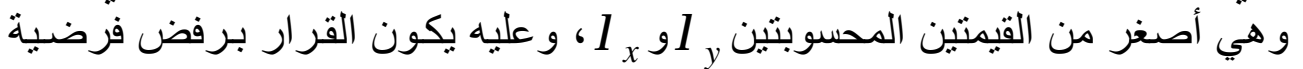

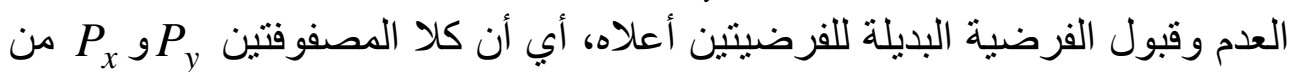

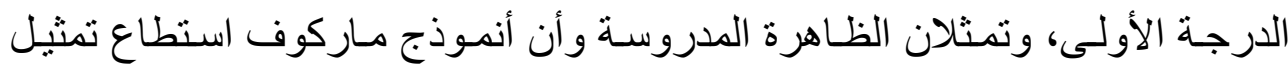
مبيعات و إير ادات الثركة من مادة اليوريا بصورة الئليدة اليدة.

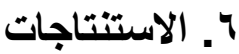

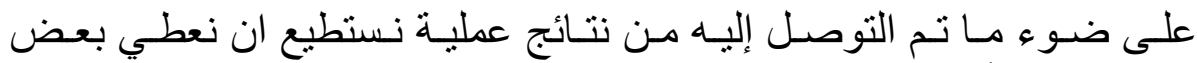

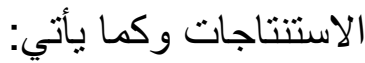

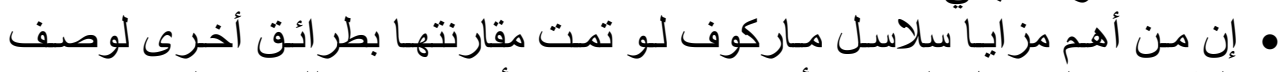

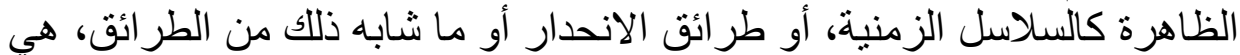

سهولة الحصول على التقدير ات مقارنة بالطر ائق أعلاه.

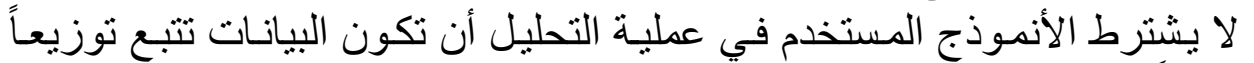

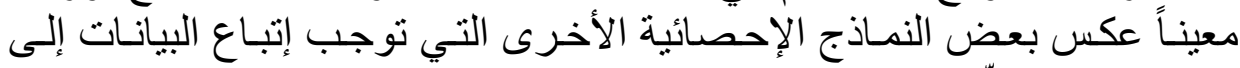
توزيع معين و إلاّ لا يتحقق التحليل.

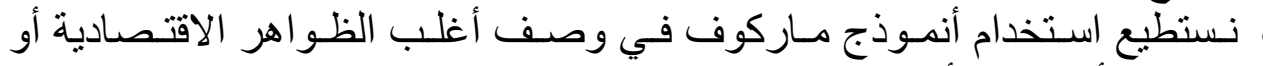

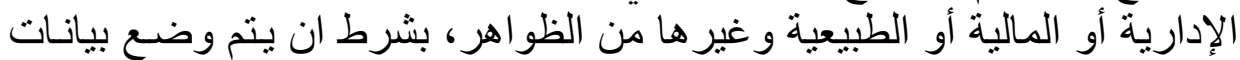

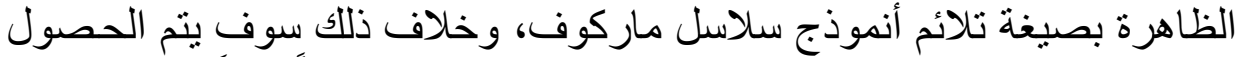

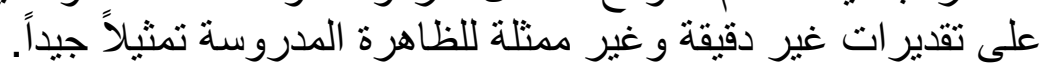

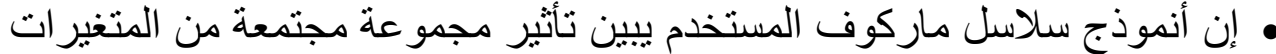

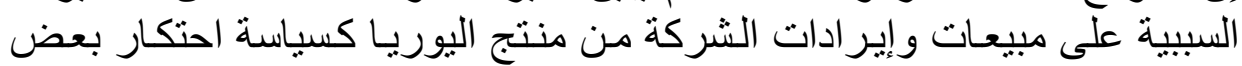

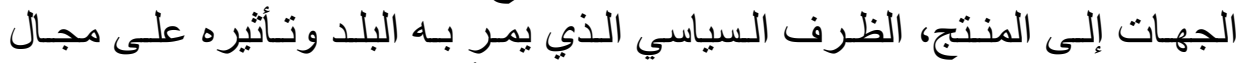

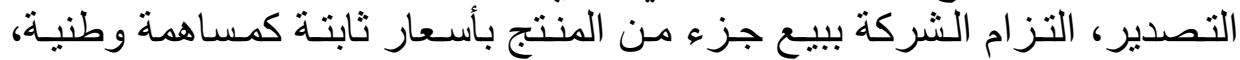

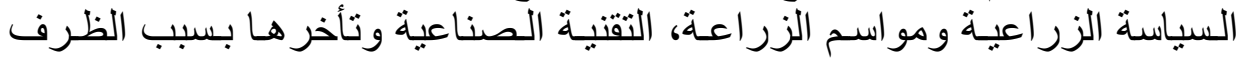

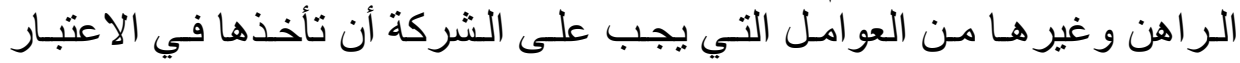
لغرض السبطرة على مبيعاتها.

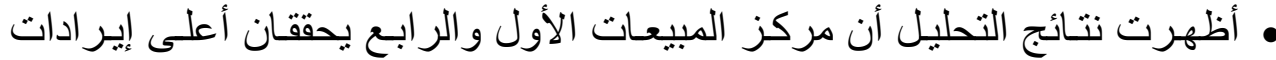

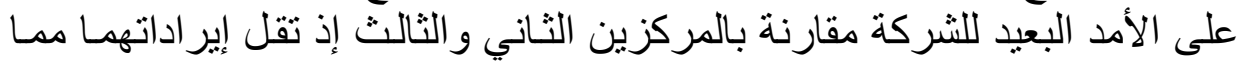

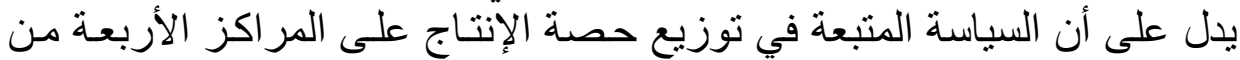
مادة اليوريا غير مربحة للشركة.

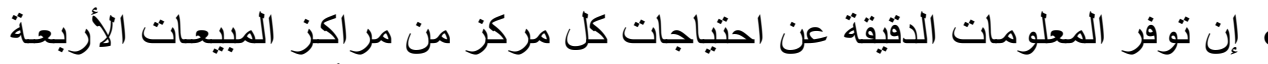

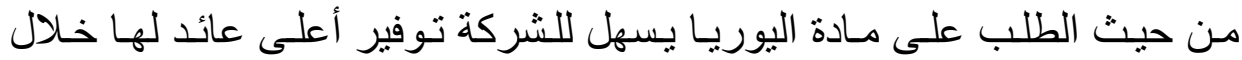
السنة، مما يسهل تجهيز تلك المر اكز بالكمية المطلوبة في الوقت المناسب. 


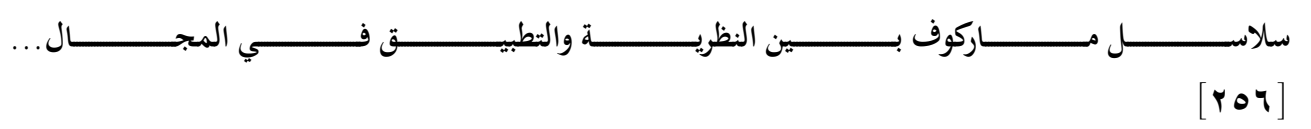

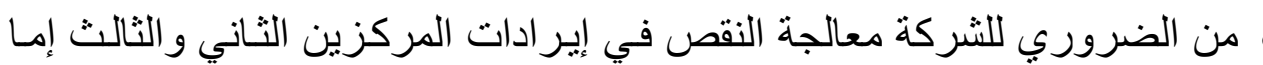

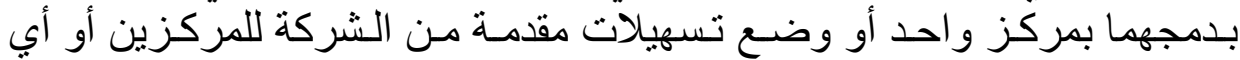
سياسة أخرى تسهم في رفع إير اداتهما.

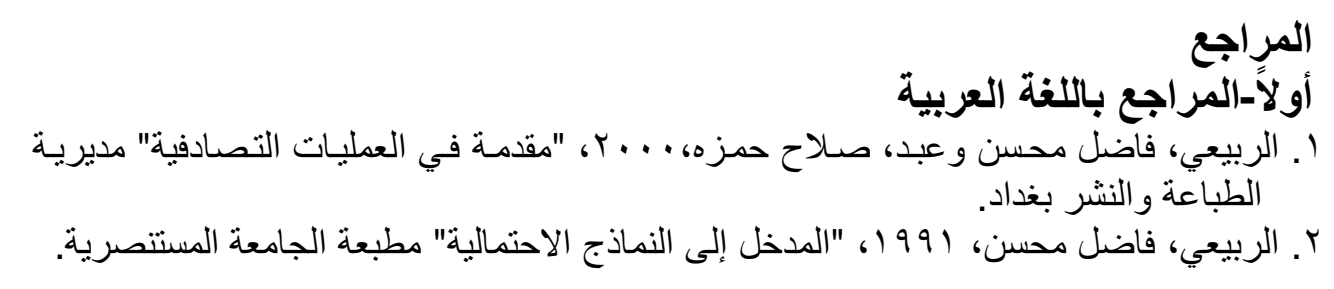

ثانياً_المراجع باللغة الأجنبية

1. Anderson, T.W. \& Goodman, L.A., 1957, "Statistical Inference About Markov Chains", Ann. Math. Stat.

2. Marius Iosifescu, 1980, "Finite Markov Processes and Their Applications", John Wiley \& Sons.

3. Norman, L. Johnson \& N. Balakrishnan, 1997, "Advance in the Theory and Practice of Statistics", John Wiley \& Sons.

4. Robert, B. \& Magdalena, N, 2000, "Probability and Statistical Inference" John Wiley $\&$ Sons. 